\title{
The VLA Frontier Fields Survey: Deep, High-resolution Radio Imaging of the MACS Lensing Clusters at 3 and $6 \mathrm{GHz}$
}

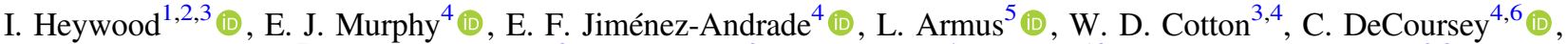 \\ M. Dickinson ${ }^{7}$ (D), T. J. W. Lazio ${ }^{8}$, E. Momjian ${ }^{9}$ (D), K. Penner ${ }^{4}$, I. Smail ${ }^{10}$ (D), and O. M. Smirnov ${ }^{2,3}$ \\ ${ }^{1}$ Astrophysics, Department of Physics, University of Oxford, Keble Road, Oxford, OX1 3RH, UK; ian.heywood@physics.ox.ac.uk \\ ${ }^{2}$ Department of Physics and Electronics, Rhodes University, P.O. Box 94, Makhanda, 6140, South Africa \\ ${ }^{3}$ South African Radio Astronomy Observatory, 2 Fir Street, Observatory, 7925, South Africa \\ ${ }^{4}$ National Radio Astronomy Observatory, 520 Edgemont Road, Charlottesville, VA 22903, USA \\ ${ }^{5}$ Infrared Processing and Analysis Center, MC 314-6, 1200 E. California Boulevard, Pasadena, CA 91125, USA \\ ${ }^{6}$ Department of Astronomy, Bryant Space Science Center, 1772 Stadium Road, Gainesville, FL 32611, USA \\ ${ }^{7}$ National Optical Astronomy Observatories, 950 N Cherry Avenue, Tucson, AZ 85719, USA \\ ${ }^{8}$ Jet Propulsion Laboratory, California Institute of Technology, 4800 Oak Grove Drive, Pasadena, CA 91109, USA \\ ${ }^{9}$ National Radio Astronomy Observatory, P.O. Box O, Socorro, NM 87801, USA \\ ${ }^{10}$ Centre for Extragalactic Astronomy, Department of Physics, Durham University, South Road, Durham DH1 3LE, UK \\ Received 2020 November 15; revised 2021 January 16; accepted 2021 January 21; published 2021 April 2
}

\begin{abstract}
The Frontier Fields project is an observational campaign targeting six galaxy clusters, with the intention of using the magnification provided by gravitational lensing to study galaxies that are extremely faint or distant. We used the Karl G. Jansky Very Large Array (VLA) at 3 and $6 \mathrm{GHz}$ to observe three Frontier Fields: MACS J0416.1 $-2403(z=0.396)$, MACS J0717.5+3745 $(z=0.545)$, and MACS J1149.5+2223 $(z=0.543)$. The images reach noise levels of $\sim 1 \mu \mathrm{Jy}$ beam $^{-1}$ with subarcsecond resolution $(\sim 2.5 \mathrm{kpc}$ at $z=3)$, providing a high-resolution view of high- $z$ star-forming galaxies that is unbiased by dust obscuration. We generate dual-frequency continuum images at two different resolutions per band, per cluster, and derive catalogs totaling 1966 compact radio sources. Components within the areas of Hubble Space Telescope and Subaru observations are cross-matched, providing host galaxy identifications for 1296 of them. We detect 13 moderately lensed $(2.1<\mu<6.5)$ sources, one of which has a demagnified peak brightness of $0.9 \mu \mathrm{Jy}_{\text {beam }}{ }^{-1}$, making it a candidate for the faintest radio source ever detected. There are 66 radio sources exhibiting complex morphologies, and 58 of these have host galaxy identifications. We reveal that MACS J1149.5+2223 is not a cluster with a double relic, as the western candidate relic is resolved as a double-lobed radio galaxy associated with a foreground elliptical at $z=0.24$. The VLA Frontier Fields project is a public legacy survey. The image and catalog products from this work are freely available.
\end{abstract}

Unified Astronomy Thesaurus concepts: Radio continuum emission (1340); Extragalactic radio sources (508); Galaxy clusters (584); Gravitational lensing (670); Very Large Array (1766)

\section{Introduction}

The Frontier Fields project was conceived around the use of the Hubble Space Telescope (HST) and Spitzer space telescope to undertake a deep-field imaging program, targeting six strong-lensing galaxy clusters, and six parallel blank fields (Lotz et al. 2017). The observations take advantage of the magnification boost provided by the foreground lensing clusters, allowing us to detect galaxies that are intrinsically very faint or at redshifts that approach the cosmic reionization epoch ( $z \sim 6$ and above). The goal of the Frontier Fields project is to gather a large sample of such sources in order to further understand star formation processes in the early universe, via measurements of the stellar mass, star formation rates, and structure of distant galaxies.

Other ground- and space-based facilities have also targeted these fields as part of the Frontier Fields campaign, and in this paper, we present the data release from a survey with the Karl G. Jansky Very Large Array (VLA). Radio observations have a singular advantage in the study of extragalactic star formation processes in that they are not susceptible to the dust obscuration that biases observational probes of star formation at ultraviolet, optical, and infrared wavelengths. Furthermore, radio is an excellent way to probe active galactic nuclei
(AGNs), due to the radio emission that can arise from the AGN core itself, as well as detecting any large-scale radio jets emanating from the region around the central supermassive black hole (Delvecchio et al. 2017). As a way to probe the obscured star formation that is present in the Frontier Field galaxies, these radio observations will form a crucial addition in pursuit of the goals of the program. This is particularly important for studies of star formation at high redshift $(z \gtrsim 3)$, as the majority of star formation in massive galaxies at such epochs takes place within dusty starburst systems, but the radio luminosities of these sources lie below the typical blank-field flux density limit of current radio facilities (e.g., Murphy et al. 2011; Magnelli et al. 2013; Wang et al. 2019; Dudzevičiūté et al. 2020).

Our observing program has targeted the three Massive Cluster Survey (Ebeling et al. 2001) Frontier Field clusters, namely MACS J0416.1-2403 $(z=0.396)$, MACS J0717.5 $+3745(z=0.545)$, and MACS J1149.5+2223 $(z=0.543$; hereafter MACS J0416, MACS J0717, and MACS J1149, respectively). These clusters were selected based on their favorable declinations for observations with both the VLA and ALMA, as well as (at the time the observations were proposed) the availability of ancillary data. The three target clusters are all 
Table 1

Coordinates and Calibrators for Each of the Three Target Clusters, as well as the On-source Integration Times for Each of the Configuration/Band Pairings

\begin{tabular}{|c|c|c|c|c|c|c|}
\hline & & MACS J0416 & MACS J0717 & MACS J1149 & Band & Configuration \\
\hline R.A. & $\mathrm{J} 2000$ & $04^{\mathrm{h}} 16^{\mathrm{m}} 08^{\mathrm{s}} .9$ & $07^{\mathrm{h}} 17^{\mathrm{m}} 34^{\mathrm{s}} .0$ & $11^{\mathrm{h}} 49^{\mathrm{m}} 36^{\mathrm{s}} .3$ & $\ldots$ & $\ldots$ \\
\hline Decl. & $\mathrm{J} 2000$ & $-24^{\mathrm{d}} 04^{\mathrm{m}} 28^{\mathrm{s}} .7$ & $37^{\mathrm{d}} 44^{\mathrm{m}} 49^{\mathrm{s}} .0$ & $22^{\mathrm{d}} 23^{\mathrm{m}} 58^{\mathrm{s}} .1$ & $\ldots$ & $\ldots$ \\
\hline Redshift & & 0.396 & 0.545 & 0.543 & $\cdots$ & $\cdots$ \\
\hline Primary calibrator & & $3 \mathrm{C} 48$ & 3C147 & $3 \mathrm{C} 286$ & $\cdots$ & $\cdots$ \\
\hline Secondary calibrator & & $\mathrm{J} 0416-1851$ & $\mathrm{~J} 0714+3534$ & $\mathrm{~J} 1158+2450$ & & \\
\hline Integration time & (hr) & 18.08 & 15.30 & 8.35 & $S$ & A \\
\hline Integration time & (hr) & 1.39 & 1.39 & 1.39 & $S$ & $\mathrm{C}$ \\
\hline Integration time & (hr) & 22.26 & 6.96 & 6.96 & $C$ & A \\
\hline Integration time & (hr) & 1.39 & 1.39 & 1.39 & $C$ & $\mathrm{C}$ \\
\hline
\end{tabular}

Note. Note that the A-configuration observations in the $S$ band for MACS J0717 include archival data previously published by van Weeren et al. (2016).

known to host diffuse radio emission associated with processes in the intracluster medium. MACS J0416 hosts a central radio halo (e.g., Ogrean et al. 2015), MACS J0717 has a very powerful central halo and a peripheral relic (e.g., van Weeren et al. 2009; Rajpurohit et al. 2021), and MACS J1149 hosts a halo as well as a candidate double relic structure (e.g., Bonafede et al. 2012).

We targeted the three clusters using the $S$ - (2-4 GHz) and $C$ band $(5-7 \mathrm{GHz})$ receivers of the VLA, with central frequencies of 3 and $6 \mathrm{GHz}$. respectively. Ninety percent of the observing was done using the most extended A configuration of the VLA to achieve the highest possible angular resolution. Coupled with long integration times, these observational parameters enable us to potentially detect and resolve $L^{*}$ galaxies at $z \sim 3$, and to discover lensed sub- $L^{*}$ galaxies at similar redshifts and above.

This paper describes the observations, data reduction, and the production and validation of the radio data products. We have also cross-matched the radio detections with the optical data from the HST (Castellano et al. 2016; Di Criscienzo et al. 2017; Shipley et al. 2018) and Subaru (Medezinski et al. 2013; Umetsu et al. 2014) to produce catalogs of radio components with host identifications and properties derived from the optical/near-infrared data, for each cluster.

An overview of the radio observations and the data calibration and imaging methods is given in Section 2. The image products and the construction of the catalogs derived from them are described in Section 3, along with a description of the optical cross-matching procedure and the estimation of gravitational lensing magnifications for radio sources that have a redshift measurement. Some results are discussed in Section 4, and Section 5 concludes the paper and provides links to the publicly available data products. The assumed cosmological model throughout this paper is $\Lambda$-CDM with $\mathrm{H}_{0}=70 \mathrm{~km} \mathrm{~s}^{-1} \mathrm{Mpc}^{-1}, \Omega_{\mathrm{M}}=0.3$, and $\Omega_{\Lambda}=0.7$.

\section{Observations, Calibration, and Imaging}

The data ${ }^{11}$ were taken with the VLA in its A (77.91 hr) and $\mathrm{C}(8.34 \mathrm{hr})$ configurations using the $S$-band $(2-4 \mathrm{GHz})$ and $C$ band $(5-7 \mathrm{GHz})$ receivers, for a total of $86.25 \mathrm{hr}$. A summary of the radio observations is provided in Table 1 . The data were taken in full polarization mode; however, this article only presents the total intensity (Stokes $I$ ) radio images and derived products.

${ }^{11}$ Project codes: 14A-012, 15A-282, and archival data from SF0858.
Each scheduling block (SB) was initially processed using the NRAO VLA pipeline. ${ }^{12}$ This is a set of scripts for the CASA (Common Astronomy Software Applications; McMullin et al. 2007) package designed to perform basic calibration steps on continuum data. The pipeline performs flagging of data due to antenna shadowing, visibility amplitudes that are exactly zero, and the initial integrations following antenna slewing. A first pass of radio frequency interference (RFI) excision from the calibrator and target scans is performed using a sliding time median filter. Following these steps, the scripts perform delay and bandpass calibration using the primary calibrators. Timedependent antenna-based complex gain corrections are then derived using the secondary calibrator and interpolated for application to the target scans. A gain correction is derived independently for each spectral window (SPW). The data were processed at the native time and frequency resolution to minimize the effects of smearing away from the phase center. Hanning smoothing was disabled in the VLA pipeline in order to minimize the effects of bandwidth smearing.

Following the execution of the pipeline, SPWs with anomalously high amplitudes were identified and discarded outright. The target field from each pointing was split into a single measurement set. The CASA mstransform task was then used to add a WEIGHT_SPECTRUM column to the visibilities, and the statwt task was used to adjust values in the WEIGHT_SPECTRUM based on the statistical properties of the visibilities for each baseline. Self-calibration did not significantly improve the noise floor in the images. The brighter sources exhibiting the worst artifacts are off-axis and likely limited by direction-dependent effects, which we did not attempt to correct for.

The target fields were imaged using the wsclean software (Offringa et al. 2014), with the image properties summarized in Table 2. Images were produced for each band and each cluster by jointly gridding and deconvolving all of the corresponding measurement sets. Large image sizes $(16,384 \times 16,384$ pixels, with pixel scales listed in Table 2) were used in order to deconvolve sources in the sidelobes of the antenna primary beam to prevent the sidelobes of the synthesized beam associated with them from affecting the target area. A Briggs (1995) robust parameter of 0.3 was used for all imaging, with additional Gaussian tapers applied to the visibilities as needed.

The spectral behavior of the sources (both intrinsic toward the beam center, and instrumentally perturbed off-axis) was captured during deconvolution by imaging the data in four

\footnotetext{
12 https://science.nrao.edu/facilities/vla/data-processing/pipeline
} 
Table 2

Summary of the Image Products Produced for Each Field

\begin{tabular}{|c|c|c|c|c|}
\hline Cluster & Image & $\begin{array}{c}\text { Angular } \\
\text { Resolution } \\
\left({ }^{\prime \prime},{ }^{\prime \prime},{ }^{\circ}\right)\end{array}$ & $\begin{array}{l}\text { Pixel } \\
\text { Scale } \\
\left({ }^{\prime \prime}\right)\end{array}$ & $\begin{array}{l}\text { rms Noise } \\
(\mu \mathrm{Jy} \\
\left.\text { beam }^{-1}\right)\end{array}$ \\
\hline \multirow{4}{*}{$\begin{array}{l}\text { MACS } \\
\text { J0416 }\end{array}$} & S-HIGH & $0.94,0.51,1.9$ & 0.16 & 1.0 \\
\hline & $S$-LOW & $4.39,3.12,43.2$ & 0.5 & 1.8 \\
\hline & $C$-HIGH & $0.53,0.3,22.7$ & 0.05 & 0.9 \\
\hline & $C$-LOW & $0.94,0.68,27.7$ & 0.12 & 1.0 \\
\hline \multirow{4}{*}{$\begin{array}{l}\text { MACS } \\
\text { J0717 }\end{array}$} & $S$-HIGH & $0.73,0.61,93.5$ & 0.16 & 0.7 \\
\hline & $S$-LOW & $3.57,3.42,79.0$ & 0.5 & 1.2 \\
\hline & $C$-HIGH & $0.33,0.27,112.7$ & 0.05 & 1.0 \\
\hline & $C$-LOW & $0.74,0.69,70.8$ & 0.12 & 1.1 \\
\hline \multirow{4}{*}{$\begin{array}{l}\text { MACS } \\
\text { J1149 }\end{array}$} & $S$-HIGH & $0.51,0.48,35.9$ & 0.16 & 0.9 \\
\hline & $S$-LOW & $3.16,3.05,101.8$ & 0.5 & 1.6 \\
\hline & $C$-HIGH & $0.28,0.27,70.9$ & 0.05 & 0.9 \\
\hline & $C$-LOW & $0.7,0.65,100.5$ & 0.12 & 1.0 \\
\hline
\end{tabular}

Note. The angular resolution is the major axis, minor axis, and position angle (east of north) of the two-dimensional Gaussian restoring beam used following deconvolution, in units of arcseconds, arcseconds, and degrees, respectively. The rms noise values are entirely consistent with the expected thermal noise values for these observations.

equal spectral chunks across the band. The approach used by wSClean during deconvolution is to find peaks in the fullband image and then deconvolve these in each subband independently. For major-cycle purposes, clean components were fitted by a second-order polynomial when predicting the visibility model. Cleaning was terminated after 100,000 iterations, which from an examination of the model and residual images was deemed to be sufficient without overcleaning. Imaging concludes with the model being restored into the full-band residual map, using a 2D Gaussian as fitted to the main lobe of the point-spread function as the restoring beam. Corrections for the conversion of brightness and flux density measurements from apparent to intrinsic values were done via the models derived from holographic measurements of the VLA primary beam by Perley (2016).

\section{Data Products}

Here we describe the radio images produced for each cluster, as well as the construction of the source catalogs and derivative images, and the verification of these products.

\subsection{Radio Images}

Four images were produced for each cluster, 12 in total. These are a high-resolution $S$-band image $(S$-HIGH), a second $S$-band image tapered to provide an approximately $3^{\prime \prime}$ synthesized beam $(S$-LOW), a full-resolution $C$-band image $(C$-HIGH), and finally, a lower-resolution $C$-band image $(C$-LOW) designed to match the angular resolution of the $S$-HIGH image. This is mainly for the determination of matched-resolution component flux densities and brightnesses, and subsequently, the determination of component spectral indices. The main properties of each image are summarized in Table 2. The $S$-HIGH images are shown in Figure 1, and Figure 2 shows contours of the $S$-HIGH image overlaid on a three-color HST image for MACS J0717. Further details on the optical catalogs are provided in Section 3.3.

\subsection{Generation of the Compact Radio Component Catalogs}

The "master" map for component extraction is taken to be the $S$-HIGH image for each cluster, as this provides both a higher surface density of detections and a larger area compared to the $C$-band images. The first step involved running the PyBDSF (Mohan \& Rafferty 2015) source finder on this image using a peak threshold of $5 \sigma$ and an island threshold of $3 \sigma$, where $\sigma$ is taken to be the source finder's own estimate of the background noise level. This is determined by stepping a box across the image and computing the standard deviation of the pixels within that region, and the resulting position-dependent values are recorded and interpolated. This produces a background noise image that is used for local estimates of $\sigma$. Peaks above five times this value are identified, and then a flood-fill method is used to delineate regions of contiguous emission down to the secondary $3 \sigma$ island threshold. Regions of significant emission are then decomposed into a series of points and Gaussian components. Default box size settings were used in each case. This procedure was performed on each of the three $S$-HIGH images.

Following this, the resulting catalogs were manually examined to remove spurious components from image artifacts. These were primarily associated with brighter sources away from the pointing center. Components belonging to sources with extended morphologies were also visually identified and placed into a separate catalog (see Section 3.7 and Appendix D). Following these steps, we obtain a catalog containing only the compact $S$-band radio sources for each cluster.

\subsection{Identifying Optical/Near-infrared Hosts}

Determining optical identifications for the radio sources relies on three existing resources for each cluster. The first two of these is a set of HST photometric catalogs created by the ASTRODEEP $^{13}$ project, by Castellano et al. (2016) for MACS J0416, and by Di Criscienzo et al. (2017) for MACS J0717 and MACS J1149, as well as the more recent catalogs from the DeepSpace project (Shipley et al. 2018). These catalogs also include spectroscopic redshifts where available, from the observing campaign by Ebeling et al. (2014). The third resource consists of the source catalogs derived from widerarea imaging with the Subaru telescope by the CLASH team, complete with photometric redshifts as presented by Medezinski et al. (2013) for MACS J0717 and Umetsu et al. (2014) for MACS J0416 and MACS J1149.

A cutout image around each compact component was created. The radio contours were overlaid on either the HST F140W image or the Subaru $z$-band image if the region was outside the HST footprint. Entries from either the ASTRODEEP HST or Subaru catalogs were overlaid on the cutout image, and visual inspection determined whether or not a cataloged optical/near-infrared association for the radio component was present, based on positional and morphological considerations. Note that we validate the astrometric alignment between radio and optical in Appendix A, and the crossmatching process is unaffected by this. In addition to verifying

\footnotetext{
13 http://www.astrodeep.eu
} 

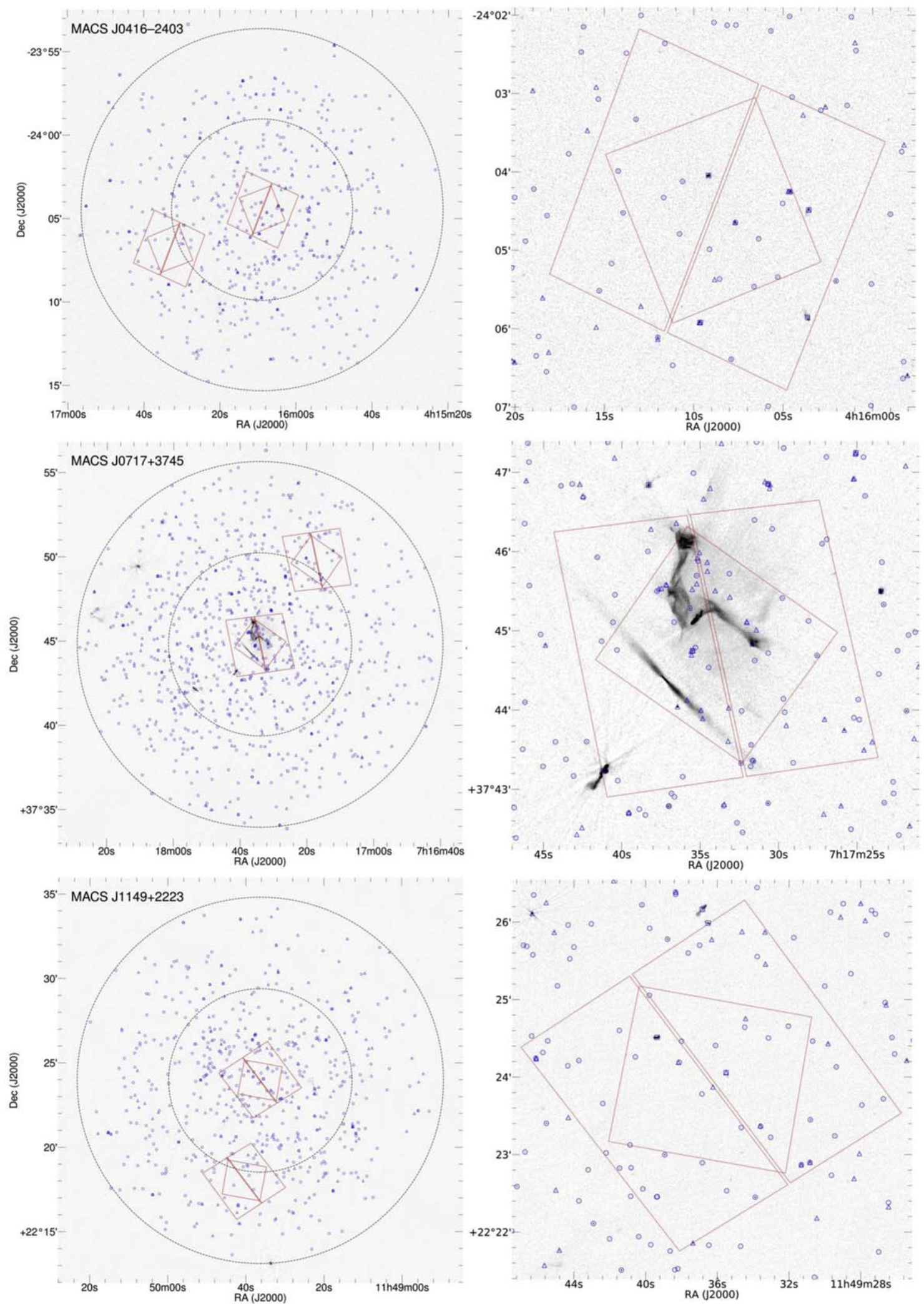

Figure 1. The $S$-HIGH images for the three clusters. The left-hand panels show the full field of view of the $S$-band observations, with the outer and inner circles showing the nominal cutoff points (at the $30 \%$ primary beam level) for the $S$ - and $C$-band images, respectively. The grayscale runs from 0.0 (white) to $40 \mu \mathrm{Jy}_{\text {beam }}{ }^{-1}$ (black) with a square-root transfer function. The inner red polygon shows the HST coverage of the cluster, and the corresponding outer marker shows the HST flanking field. The right-hand panels in the figure zoom in on the HST cluster area. The blue markers on the figure show cataloged radio sources. Circular markers represent compact radio sources that have a host galaxy identified in either the HST or Subaru data. Triangular markers represent compact radio sources for which a cataloged optical/near-infrared host has not been identified. Square markers represent extended morphology radio sources, plotted at the location of the cataloged counterpart from the HST or Subaru observations. Please refer to Section 3.3 for details of the cross-matching process. 


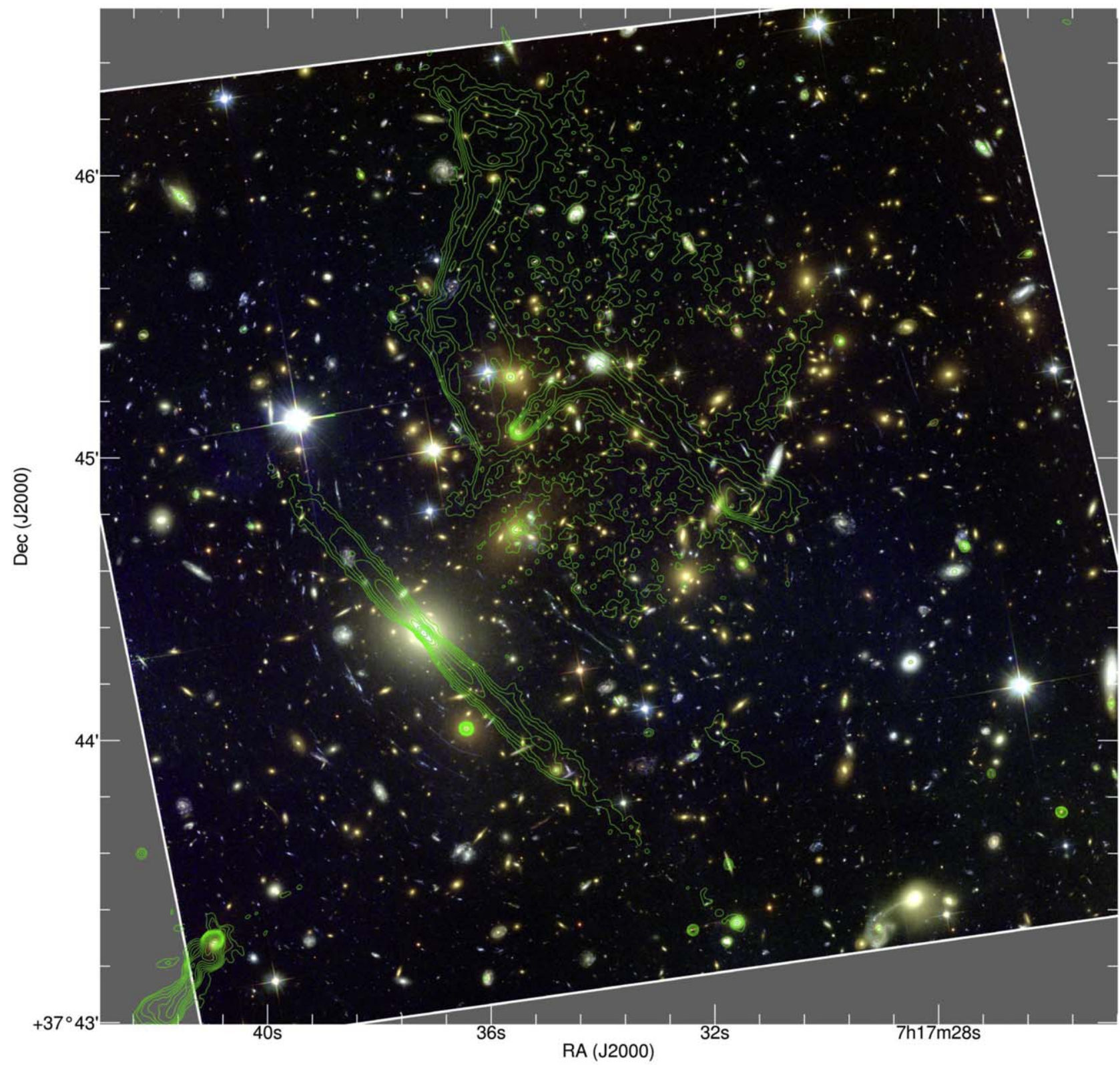

Figure 2. The S-HIGH image for MACS J0717, shown as a contour plot overlaid on a three-color image formed from the F814W, F606W, and F435W filters from the HST observations. The optical counterparts to numerous compact and extended radio sources are visible. The extended structures associated with the bright radio relic in this cluster dominate the radio emission. The base contour level is $1.6 \mu \mathrm{Jy}$ beam ${ }^{-1}$, and from there the contour increments are $1.2 \times \sqrt{3}^{n} \mu \mathrm{Jy}$ beam ${ }^{1}$ where $(n=0,1,2,3 \ldots)$.

potential hosts for the radio components, manually inspecting each of the compact components also allowed flags to be applied to sources that (i) could be well represented by single Gaussian components but had been decomposed into several components by PyBDSF, and (ii) had significantly extended structures associated with them that had been missed in the first pass. Components belonging to the first category were force-fit with a single Gaussian using the CASA imfit task, and the multiple entries belonging to this source in the component catalog were replaced by this single component. Radio components belonging to the second category were removed from the compact catalog and placed into the extended catalog. Note that this process only cross-matched radio components with cataloged optical sources from the HST or Subaru catalogs.
At this stage, the compact $S$-band catalog for each cluster exists in two subsets: those with optical/near-infrared matches (the "radio-optical" catalog) and those without (the "radio" catalog). Relevant properties derived from the HST observations (e.g., optical IDs, positions, stellar mass and star formation rate estimates, spectroscopic and photometric redshifts) are merged with the radio-optical catalog, using the more recent catalogs from the DeepSpace project (Shipley et al. 2018), using positional coincidence matches between these catalogs and the ASTRODEEP catalogs. Additional entries present in the DeepSpace catalogs that were not visually matched as part of the ASTRODEEP process were assigned to radio components by conducting nearest-neighbor crossmatching for the additional sources that the DeepSpace catalogs contain. Following, e.g., Ivison et al. (2007), the 
match radius was set according to

$$
\Delta_{\text {R.A. }}=\Delta_{\text {Decl. }}=0.66 \frac{\theta}{\mathrm{S} / \mathrm{N}},
$$

which predicts how $\Delta_{\text {R.A. }}$ and $\Delta_{\text {Decl. }}$ (the scatter in R.A. and decl. of the radio component) scale with the signal-to-noise ratio $(\mathrm{S} / \mathrm{N})$ of the detection for a synthesized beam with an FWHM of $\theta$. Evaluating Equation (1) for $\mathrm{S} / \mathrm{N}=5$ for the $S$-HIGH images (according to the noise values reported in Table 2) results in match radii of $0 . " 22,0$ !" 16 , and 0 !" 12 for MACS J0416, MACS J0717, and MACS J1149, respectively. Note that prior to cross-matching, the offsets described in Appendix A were removed to further align the cataloged positions. A complete description of the columns for both the radio-optical and the radio catalog, including the properties derived in the sections that follow, is provided in Appendix C. Stellar masses, star formation rates, and specific star formation rates from DeepSpace are included in the radio-optical catalog where appropriate. For a detailed analysis of radio versus optical star formation rates, we refer the reader to the companion paper to this work by Jiménez-Andrade et al. (2021).

\subsection{Resolved Sources, Uncertainties in the Deconvolved Sizes, and the "Best" Flux Density Estimates}

Although PyBDSF provides the deconvolved source sizes, the associated uncertainties in the major and minor, and position angles that are returned are the same as the apparent sizes in the map plane (at the time of writing). We thus estimate the intrinsic source (major axis) sizes and the associated uncertainties according to the method presented in Appendix B.

In addition to the component peak brightnesses and integrated flux densities measured by the source finder, we also provide a "best" flux density estimate $\left(S_{*}\right)$ based on an assessment of how reliably resolved the Gaussian components are. This largely follows the method adopted by Murphy et al. (2017), modified for the considerations above that cater for the fact that the restoring beam in our maps was not forced to be circular. If the criterion

$$
\phi^{\prime}-\theta_{\text {beam }}^{\prime} \geqslant 2 \sigma_{\phi}^{\prime}
$$

is satisfied, then we deem a source to be reliably resolved, where $\theta^{\prime}$ is the FWHM of the elliptical beam as projected along the source major axis, and $\phi^{\prime}$ and $\sigma_{\phi}^{\prime}$ are the source major axis and associated uncertainty as measured by PyBDSF. In this case, $S_{*}$ is set to the integrated flux density of the component as determined by the source finder. For sources that are marginally resolved (those that do not satisfy the Equation (2) criterion), we set $S_{*}$ to be the geometric mean of the integrated flux density and peak brightness. The uncertainties in these two quantities are anticorrelated when fitting Gaussians, and hence, the geometric mean provides the best flux density measurement for a Gaussian fit to a faint component (Condon 1997).

\subsection{Adding $6 \mathrm{GHz}$ Information and Determining Spectral Indices}

The PyBDSF source finder was also run on the $C$-LOW images, and the results were cross-matched with the "radiooptical" and "radio" catalogs described above. The $C$-LOW image matches the $S$-HIGH images in terms of angular resolution, making for more robust flux density comparisons for compact features between the two frequencies, and thus for more reliable spectral index estimates. All components in the $S$-band catalog that had a $C$-band match within a radius of $1^{\prime \prime}$ were visually examined (via $S$-band contours over the $C$-band image) to confirm that the $C$-band component was neither spurious nor fragmented into multiple components. Genuine $C$-band matches were then associated with the relevant $S$-band components. Spectral index $(\alpha)^{14}$ estimates for compact components were derived from the dual-frequency "best" flux density measurements $\left(S_{*}\right)$ and added to the catalog.

\subsection{Lensing Magnifications}

The Frontier Fields have publicly available lensing models, ${ }^{15}$ derived independently by five different teams. Further details are given by Lotz et al. (2017), with the mapping teams making use of data from Schmidt et al. (2014), Vanzella et al. (2014), Diego et al. (2015), Merlin et al. (2015, 2016), Castellano et al. (2016), Jauzac et al. (2016), Kawamata et al. (2016), Limousin et al. (2016), and Caminha et al. (2017).

The models take the form of images that capture the spatial variation in mass surface density $(\kappa)$ and lensing shear $(\gamma)$ over each cluster. The magnification $(\mu)$ for a point-like source at a given redshift $\left(z_{s}\right)$ behind the lensing cluster (at redshift $z_{l}$ ) can be modeled using these two parameters via the relationship

$$
1 / \mu=\left(1-\kappa_{z}\right)^{2}-\gamma_{z}^{2}
$$

where $\kappa_{z}$ and $\gamma_{z}$ are the model values for mass surface density and shear scaled by the ratio $D_{l s} / D_{s}$, where $D_{l s}$ is the angular diameter distance between the cluster and the source, and $D_{s}$ is the angular diameter distance from redshift zero to the source.

Magnification estimates were determined for radio components that have either spectroscopic or photometric redshifts by evaluating Equation (3), using the source redshift estimate. The preferred order of redshifts when calculating lensing is (i) spectroscopic redshifts from the DeepSpace catalogs, (ii) photometric redshifts from the DeepSpace photometry fitting as derived using the EAZY code (Brammer et al. 2008), and (iii) Bayesian photometric redshifts from the CLASH catalogs. This process was repeated for each lensing model, and the median magnification over all available models is evaluated and recorded in the catalog. Median absolute deviations of $\mu$ across all available models are also tabulated in order to provide some measure of the magnification uncertainty. Note that when extracting the values from the maps of $\kappa$ and $\gamma$, the position of the radio component was used, not the position of the optical host, naturally leading to some differences between the magnifications in the radio-optical catalog and those derived from the DeepSpace catalogs. Note that we do not track inverted parity $(\mu<0)$ sources in the catalog as we only present the median absolute value over all available lensing models.

\subsection{Extended Radio Sources}

As mentioned in Section 3.2, the visual confirmation of optical hosts in conjunction with the component models

\footnotetext{
${ }^{14}$ Here, we define the flux density $S$ being related to frequency $\nu$ ) via the spectral index $\alpha$ according to $S \propto \nu^{\alpha}$.

${ }^{15}$ https://archive.stsci.edu/prepds/frontier/lensmodels/
} 
derived by PyBDSF allowed a thorough identification of radio sources with extended morphologies. These features were subdivided into three categories: (i) extended radio sources with cataloged optical hosts, (ii) extended radio sources with no cataloged optical host, and (iii) diffuse structures (e.g., relics and halos) that are likely arising due to processes in the foreground clusters. Optical IDs were associated with the first category via the same method as for the compact components, and categories (i) and (ii) were distinguished from (iii) using morphological and optical-ID considerations.

In order to determine the integrated flux density of the extended sources, we employ the ProFound software package (Robotham et al. 2018). Designed primarily with large optical surveys in mind, the software has been found to provide superior photometric estimates to other common source-finding packages when applied to both simulated and real radio interferometer data (Hale et al. 2019). ProFound does not perform component fits to regions of extended emission, instead adopting an approach whereby thresholded regions are iteratively dilated until the surface brightness measurement converges.

Cutout images for each extended region from both the $S$-LOW and $S$-HIGH images were processed using ProFound, the former also being used to better gauge the total integrated flux density for sources that may be partially resolved out by the A-configuration observations. The resulting catalogs from ProFound were pruned of any measurements of additional sources in the field in order to only derive properties from the extended source being considered.

Optical/near-infrared and radio contour composite images, notes on individual extended sources, and their tabulated radio properties are presented in detail in Appendix D. Note that the well-studied foreground Fanaroff-Riley Type-1 (FR-I) source in the MACS J0717 field and the narrow-angle-tail (NAT) galaxy embedded in the radio halo are not included.

\section{Results}

\subsection{Redshift Distributions of Radio Sources}

There are 1262/55 radio sources with a photometric/ spectroscopic redshift across the three fields, including the host galaxies of radio sources with complex morphologies. The distribution of these redshifts is shown in Figure 3, with photometric measurements shown in gray and spectroscopic measurements shown in red. The median redshift measured from the cataloged sources from all three clusters is 0.88 . This is lower than generally reported from other radio-selected deepfield studies (e.g., Smolčić et al. 2017); however, the overdensities of galaxies in the clusters themselves will pull this median value down. On a per-cluster basis, the median redshifts are 0.9, 0.75, and 1.03 for MACS J0416, MACS J0717, and MACS J1149, respectively. If we exclude sources within \pm 0.05 of the cluster redshift, the median redshifts of the radio-selected objects become $0.92,0.85$, and 1.13 , having excluded 10, 58, and 38 objects from MACS J0416, MACS J0717, and MACS J1149, respectively. There appear to be significantly more sources out to $z \sim 1.5$ in MACS J1149, and the detections in this field appear to be less clustered along the line of sight (see also Figure 7). Despite the limiting magnitudes for the Subaru observations being the same, the surface density of strongly lensed sources varies by a factor of $>2$ across the three fields (Umetsu et al. 2014). This suggests
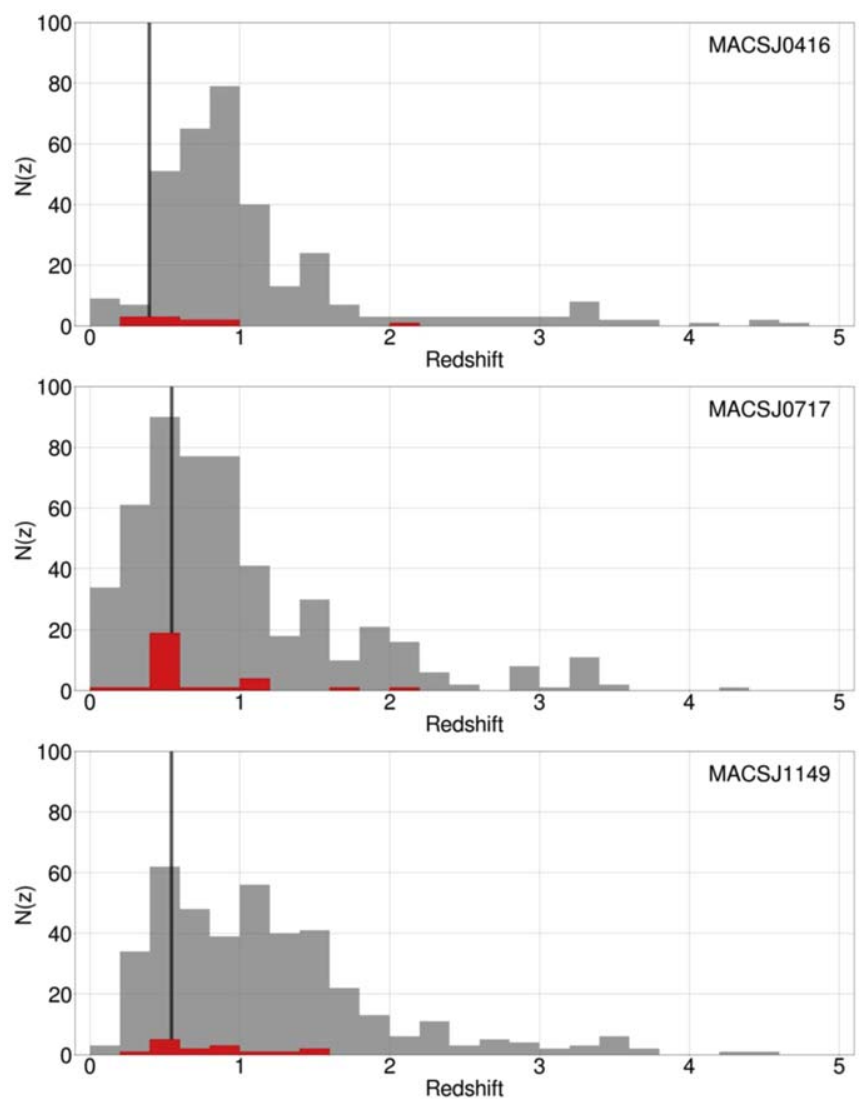

Figure 3. Distribution of the spectroscopic (red) and photometric (gray) redshifts as measured from the optical IDs of the radio components detected in this survey. This includes the host galaxies of extended radio structures. The median redshift measured across all three clusters is 0.88 . The vertical lines on each plot mark the redshifts of the clusters.

that sample variance is the likely explanation for the boxy (or possibly bimodal) redshift distribution of the radio sources in MACS J1149.

\subsection{Spectral Indices of Compact Radio Sources}

Accurate radio spectral index $(\alpha)$ measurements are essential for deriving rest-frame quantities such as spectral luminosity and, by extension, parameters such as star formation rates (e.g., Jiménez-Andrade et al. 2021). The use of measured values of $\alpha$ as opposed to adopting characteristic or canonical values (typically assumed to be -0.7 to -0.8 for synchrotron emission) can significantly reduce the scatter and biases in studies involving the radio/far-infrared correlation, as demonstrated by Gim et al. (2019), who also demonstrate the importance of deriving values of $\alpha$ from dual-frequency images that are resolution-matched.

Figure 4 shows the $3 \mathrm{GHz}$ integrated flux densities of the compact radio sources against their $3-6 \mathrm{GHz}$ spectral index. Note that the plot only shows sources that have a $C$-band detection within the $50 \%$ gain region of the VLA $C$-band primary beam at $6 \mathrm{GHz}$ (for a total of 169 objects). Within this region, both the $C$-band and especially $S$-band primary beam responses do not impart significant attenuation, and thus, the selection biases introduced by the frequency-dependent antenna primary beam pattern over the broad bandwidths of both observing bands are reduced. Selection biases persist, however, introduced by the unknown distribution of source 


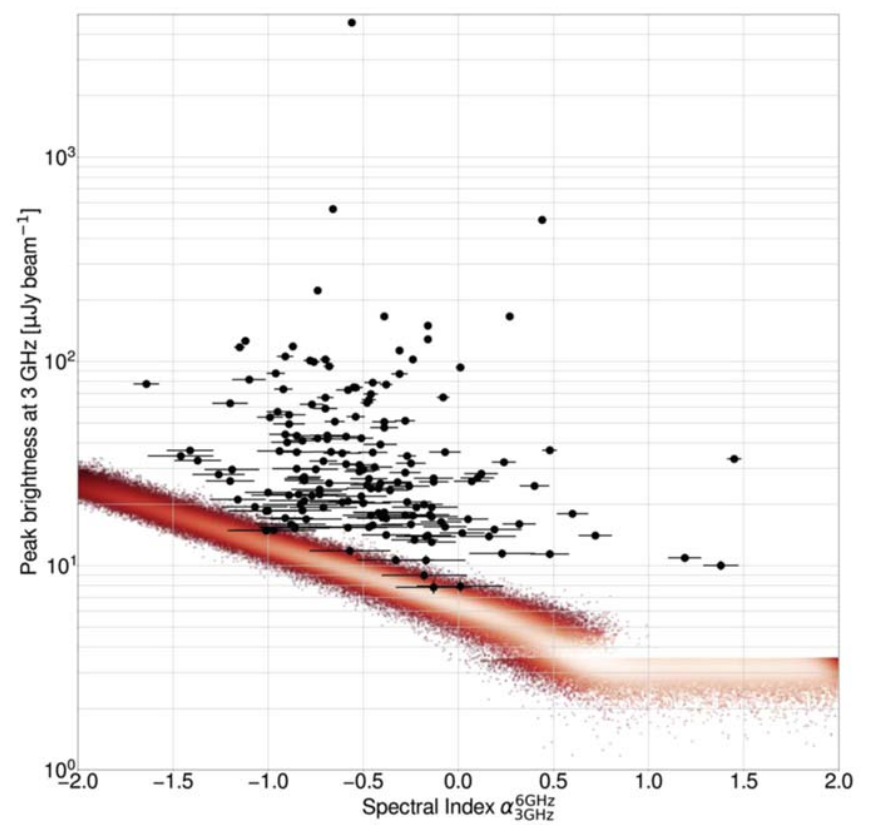

Figure 4. Integrated flux densities of the $S$-band radio components against their 3-6 GHz spectral index value. The cloud of points shows the results of a Monte Carlo simulation used to gauge the selection function in this plane. Note that this is a population of simulated sources that are nondetections. Sources below this distribution should be undetectable, and our catalog is only complete for $-2<\alpha<2$ for peak $3 \mathrm{GHz}$ brightnesses above $30 \mu \mathrm{Jy} \mathrm{beam}^{-1}$. Please see Section 4.2 for details.

spectral indices and the slightly different depths of the $S$-HIGH and $C$-LOW images, which are used to measure $\alpha$ for the cataloged sources.

We investigate the selection function imposed on the $S-\alpha$ plot using a Monte Carlo simulation. The spectral index range -2 to +2 is partitioned into 400 bins (width 0.01 ). For each of these bins, a source with the corresponding spectral index is created and assigned a peak brightness at $3 \mathrm{GHz}$. The corresponding peak brightness at $6 \mathrm{GHz}$ is calculated according to the assigned value of $\alpha$. Both the 3 and $6 \mathrm{GHz}$ values are then perturbed with noise drawn from a Gaussian distribution with a mean of zero and a standard deviation corresponding to the lowest $S$-HIGH $\left(0.7 \mu \mathrm{Jy}\right.$ beam $\left.^{-1}\right)$ and $C$-LOW $(1.0 \mu \mathrm{Jy}$ beam $^{-1}$ ) rms noise values across the three clusters, as listed in Table 2. If the noisy simulated brightness measurements at 3 and $6 \mathrm{GHz}$ exceed five times the rms noise values in both bands, then the simulated source is considered to be detected and in the real-world case would have a legitimate $\alpha$ measurement in the catalog. Because we are interested in the sources we may be missing from Figure 4, the above procedure is repeated with a steadily declining $3 \mathrm{GHz}$ brightness. When the simulated source does not meet the peak brightness detection criteria, i.e., either the 3 or $6 \mathrm{GHz}$ brightness drops below the threshold, the source is considered undetected and the peak brightness and $\alpha$ values are noted. This process is repeated 400 times for all $400 \alpha$ bins, and the resulting distribution of simulated sources that have dropped below the detection threshold is plotted as the red cloud of points in Figure 4, where the density of sources increases from dark red to white.

The fact that there is a detection criterion at both 3 and $6 \mathrm{GHz}$, but the plot shows only the $3 \mathrm{GHz}$ measurements, imparts two distinct regions to this distribution. Sources to the

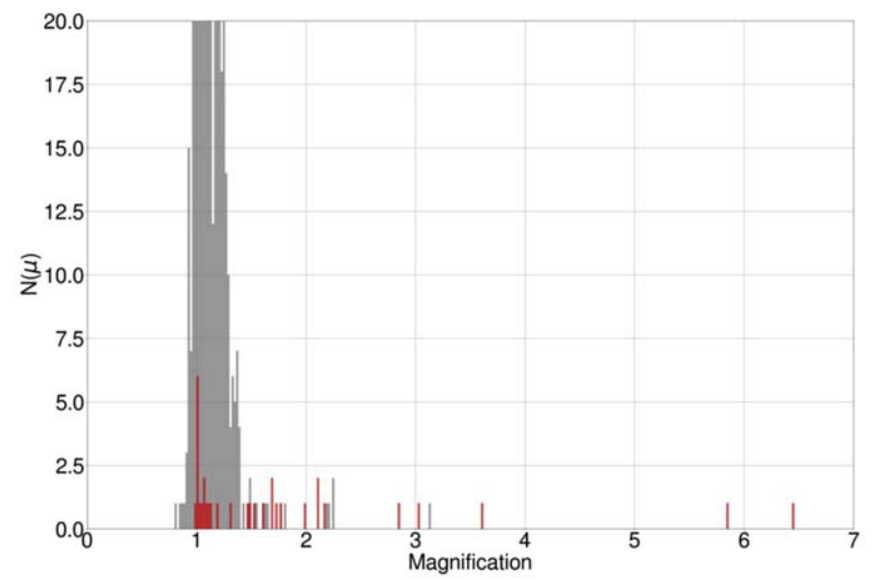

Figure 5. Distribution of the lensing magnifications as derived in Section 3.6 for sources with spectroscopic redshifts (red) and photometric redshifts (gray). The bin widths are 0.2 . The bins in the 1.0-1.2 range run over the figure limit, dominated by those for which a unity magnification is entered into the catalog as they are beyond the regions covered by the lensing models. Several sources are demagnified $(0<\mu<1)$. Note that we do not track inverted parity $(\mu<0)$ sources.

left of the distribution have steep spectra that render them too faint for detection at $6 \mathrm{GHz}$, but the corresponding full range of noisy $3 \mathrm{GHz}$ brightness measurements is present. Sources to the right of the distribution have inverted spectra that cause them to drop below the $3 \mathrm{GHz}$ detection threshold, delineated by the hard upper limit on the right of the distribution. Essentially we are seeing the manifestation of Eddington bias as it applies to a combination of two peak brightness-limited samples. Our spectral index selection function is thus only complete for $-2<\alpha<2$ above a peak $3 \mathrm{GHz}$ brightness of $30 \mu \mathrm{Jy}_{\text {beam }}{ }^{-1}$.

The median spectral index of the 169 objects plotted in Figure 4 is -0.5 ; however, for the 74 sources with peak brightnesses above $30 \mu \mathrm{Jy}$ beam $^{-1}$ at $3 \mathrm{GHz}$, the median value of $\alpha$ is -0.63 . For the canonical synchrotron radiation spectral index of -0.7 , our peak $S$-band completeness limit is equal to $50 \mu \mathrm{Jy}_{\text {beam }}{ }^{-1}$ at $1.4 \mathrm{GHz}$. Our median value is consistent with previous dual-band studies at comparable depths (e.g., Huynh et al. 2015; Gim et al. 2019; Huynh et al. 2020), as well as inband measurements made using the $1-2 \mathrm{GHz} L$-band receivers of the VLA (Heywood et al. 2016, 2020). We note also that the dual-band studies involving measurements at $\sim 5 \mathrm{GHz}$ and above also revealed a significant fraction of flat and inverted spectrum sources in addition to those with typical synchrotron spectra, something that is also evident in Figure 4.

\subsection{Lensed Compact Radio Sources}

Figure 5 shows a histogram of the lensing magnification estimates, the derivation of which is given in Section 3.6. As with Figure 3, sources with spectroscopic redshifts are marked in red. We detect 13 objects with magnification factors greater than 2, 7 of which have spectroscopic redshifts. Figure 6 shows the cutouts for each of these sources. The ID, redshift, and median lensing magnification are given above and on the numbered panel for each source. The background of each image is a three-color rendering of the F814W, F606W, and F435W HST filters overlaid with the $S$-HIGH contours, with contour levels provided in the caption. 
VLAHFF-J041606.36-240451.2

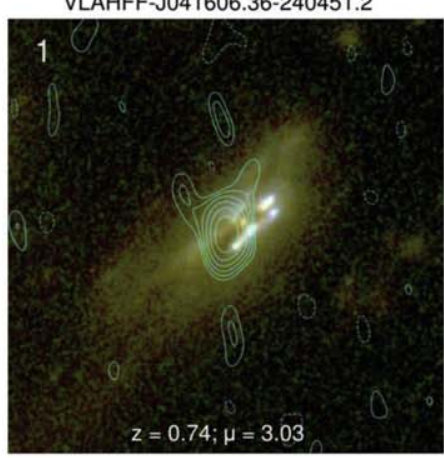

VLAHFF-J071725.85+374446.2

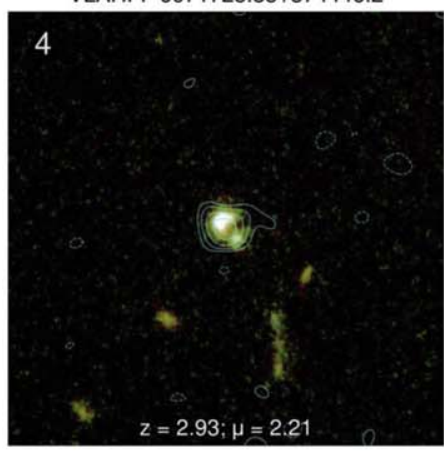

VLAHFF-J041606.62-240527.8

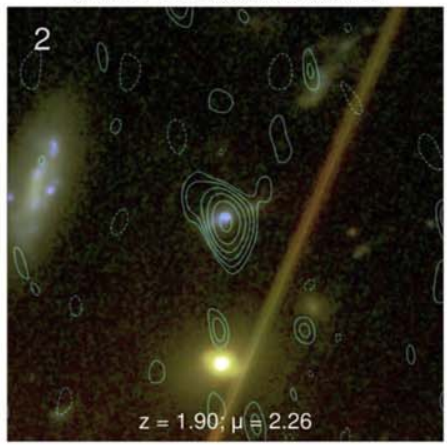

VLAHFF-J071730.65+374443.1

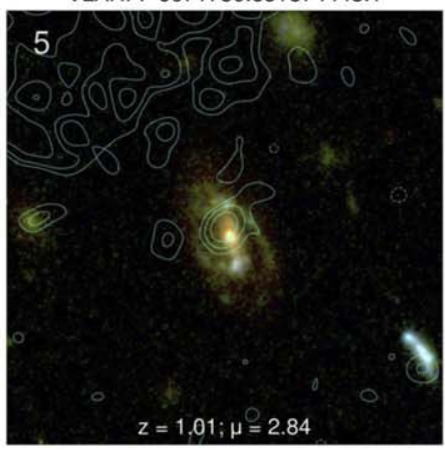

VLAHFF-J041611.67-240419.6

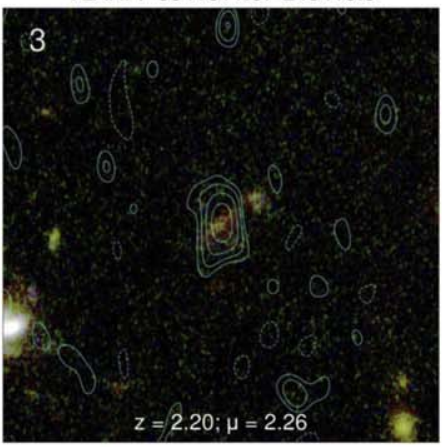

VLAHFF-J071733.14+374543.2

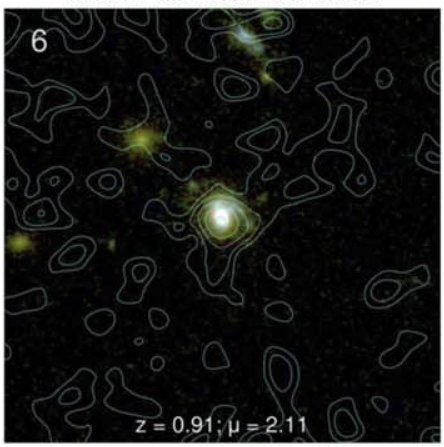

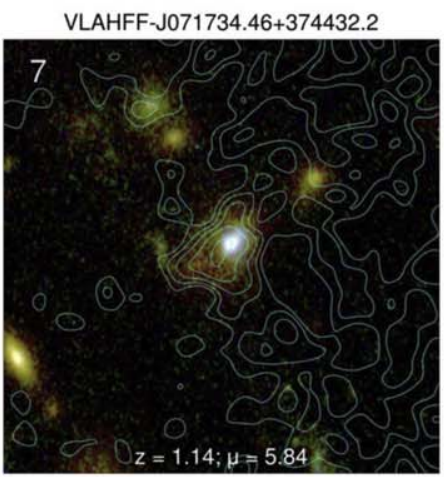

VLAHFF-J071735.22+374541.7

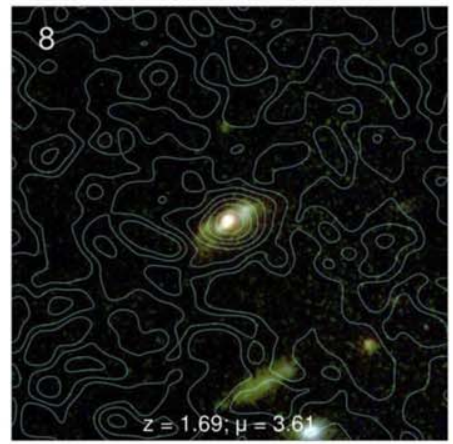

VLAHFF-J071736.66+374506.4

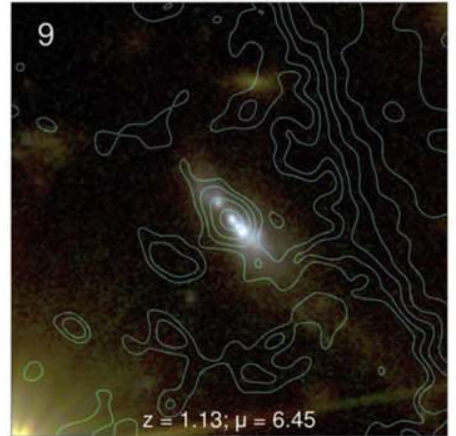

VLAHFF-J071740.55+374506.4

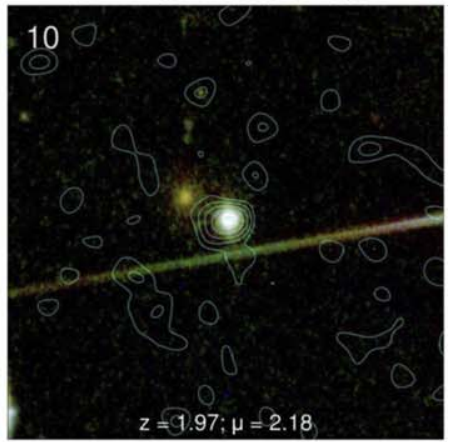

VLAHFF-J114932.03+222439.3

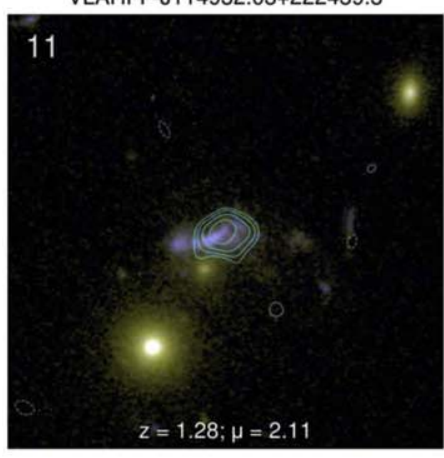

VLAHFF-J114934.46+222438.5

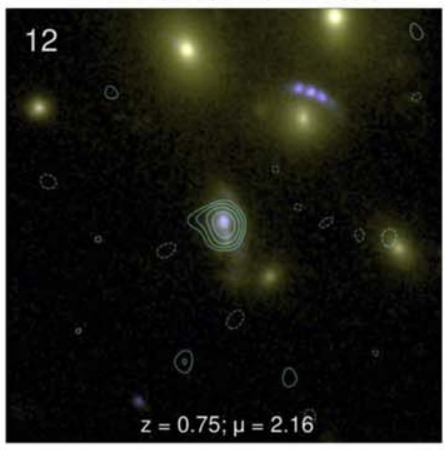

VLAHFF-J114936.09+222424.4

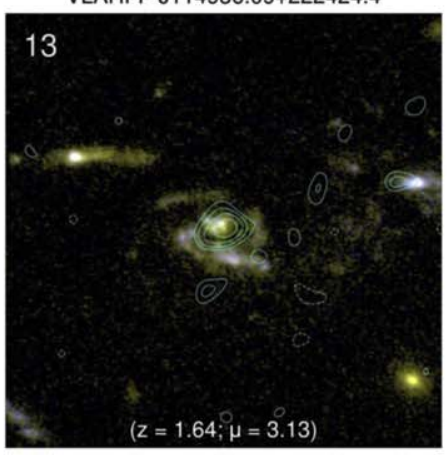

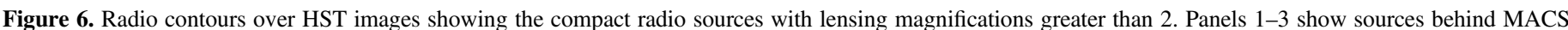

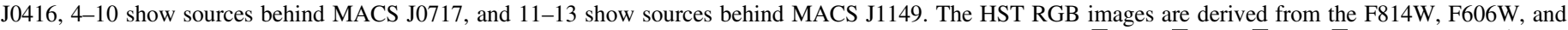

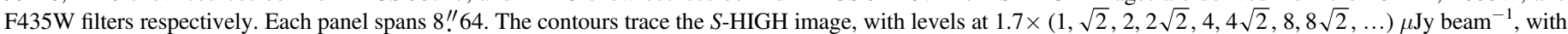

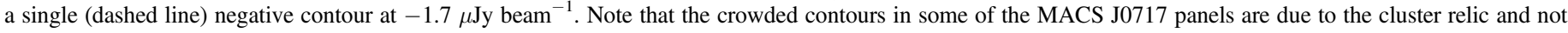

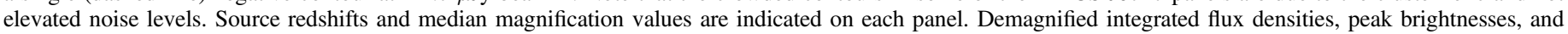
effective noise levels for each source are listed in Table 3. 
Table 3

Magnifications and Demagnified Integrated Flux Densities, Peak Brightnesses, and Effective Noise Levels for the 13 Lensed Sources Presented in Figure 6

\begin{tabular}{|c|c|c|c|c|c|}
\hline Panel & ID & $\mu$ & $\begin{array}{c}S_{\text {int }}^{\text {demagnified }} \\
(\mu \mathrm{Jy})\end{array}$ & $\begin{array}{c}\begin{array}{c}S_{\text {peak }}^{\text {demagnified }} \\
\left(\mu \mathrm{Jy} \mathrm{beam}^{-1}\right)\end{array}\end{array}$ & $\begin{array}{c}\sigma^{\text {demagnified }} \\
\left(\mu \mathrm{Jy} \text { beam }^{-1}\right)\end{array}$ \\
\hline 1 & VLAHFF-J041606.36-240451.2 & 3.03 & 6.71 & 4.76 & 0.33 \\
\hline 2 & VLAHFF-J041606.62-240527.8 & 2.26 & 7.75 & 5.06 & 0.44 \\
\hline 4 & VLAHFF-J071725.85+374446.2 & 2.21 & 4.04 & 3.03 & 0.41 \\
\hline 5 & VLAHFF-J071730.65+374443.1 & 2.84 & 2.44 & 1.85 & 0.32 \\
\hline 6 & VLAHFF-J071733.14+374543.2 & 2.11 & 8.43 & 3.1 & 0.43 \\
\hline 9 & VLAHFF-J071736.66+374506.4 & 6.45 & 1.96 & 0.9 & 0.14 \\
\hline 10 & VLAHFF-J071740.55+374506.4 & 2.18 & 4.17 & 3.78 & 0.41 \\
\hline 11 & VLAHFF-J114932.03+222439.3 & 2.11 & 9.99 & 3.04 & 0.43 \\
\hline 12 & VLAHFF-J114934.46+222438.5 & 2.16 & 6.05 & 3.61 & 0.42 \\
\hline 13 & VLAHFF-J114936.09+222424.4 & 3.13 & 4.26 & 2.11 & 0.29 \\
\hline
\end{tabular}

Note. The first column refers to the panel number for each source in that figure.

The majority of these sources are blue star-forming galaxies with spiral or disturbed morphologies and prominent dust lanes. The mean redshift is 1.73 (standard deviation 0.81 ). For each of the radio components shown in Figure 6, we provide demagnified, intrinsic values for the integrated flux density, peak brightness, and effective noise levels in Table 3. The source with the highest magnification factor $\left(\mu_{\text {median }}=6.45\right)$ is VLAHFF-J071736.66+374506.4, which has an intrinsic peak brightness of $0.9 \mu \mathrm{Jy}$ beam $^{-1}$ at an effective noise level of $140 \mathrm{nJy}$ beam $^{-1}$. This makes it a candidate for the faintest radio source detected to date (see also Jackson 2011). The total star formation rate for this source is modest, at $10 M_{\odot} \mathrm{yr}^{-1}$ (for more details, see Jiménez-Andrade et al. 2021).

We note some differences between our lensed source catalog and that of van Weeren et al. (2016). The principal reason for this is our use of median rather than mean magnifications. The former is far more robust to strong outliers that may be present in the range of lensing models used. For example, when using mean magnifications for our catalogs, one source had an average magnification factor of 150 . Examination of the nine individual magnification values from each of the models revealed one model predicting a magnification factor of 1305.8 , whereas the median of the other eight values was 6.22 . The use of median values also results in fewer sources with $\mu>2$.

\subsection{Demagnified $3 \mathrm{GHz}$ Radio Luminosities and the Discovery of a Powerful Radio Galaxy at $\mathrm{z}>4$}

Here we bring together the results of Sections 4.1-4.3 to compute the demagnified $3 \mathrm{GHz}$ radio luminosities for the compact radio components. These are plotted against redshift for each cluster in Figure 7. The vertical lines show the redshifts of the foreground clusters in each panel, and the dashed line shows the $5 \sigma$ detection limit based on the noise measurements of the $S$-HIGH images as listed in Table 2. Measured spectral index values are used where available, and our median value of -0.63 is used otherwise. The preferential order for redshifts is as before, namely (i) DeepSpace spectroscopic redshifts, (ii) DeepSpace photometric redshifts, and (iii) CLASH photometric redshifts. As elsewhere, the last dominate the counts. The magnification corrections demonstrate that we are detecting high-redshift galaxies below the formal detection threshold, although the extreme outlier visible in the MACS J0717 field is likely the result of an improper photometric redshift fit. Figure 7 also shows evidence for more distant clustering in redshift along the line of sight, particularly in the MACS J0416 field.

The MACS J0416 luminosity plot reveals a source at $z=4.06$ (VLAHFF-J041559.99-240132.5), with a $3 \mathrm{GHz}$ restframe luminosity of $4.1 \times 10^{25} \mathrm{~W} \mathrm{~Hz}^{-1}$. The $3-6 \mathrm{GHz}$ spectral index of this source is steep (or ultra-steep by some definitions, $\alpha=-1.13 \pm 0.05$ ). This is a characteristic known to be an effective method to search for powerful radio sources at high redshift, although the physical explanation is not conclusively understood (e.g., Singh et al. 2014). The source is deemed to be resolved, although it is well characterized by a single Gaussian component, and there is no evidence for large-scale jet emission in either the $S$-HIGH or $S$-LOW images. This source is thus likely a newly discovered powerful high- $z$ radio galaxy and worthy of follow-up observations.

\subsection{Intrinsic Radio Sizes}

The $C$-HIGH images with angular resolutions of $\sim 0$ " $3-0$ ". 5 will offer the best means for estimating intrinsic source sizes from this project. To examine this, we present the distribution of the FWHM deconvolved source major axes in the upper panel of Figure 8 . The median deconvolved source size $\left(\theta_{\mathbf{M}}\right.$; for entries in the catalog that have nonzero values) is 0 ". $27 \pm 0$ ". 25 . For the assumed cosmology, the radio components with associated redshift measurements have a median physical size of $1.9 \pm 1.2 \mathrm{kpc}$. The corresponding distribution in physical units is shown in the lower panel of Figure 8.

Higher angular resolution observations reaching $\sim 0.6 \mu \mathrm{Jy}$ beam $^{-1}$ have been made of the GOODS North field at the $X$ band (10 GHz; Murphy et al. 2017). This study also used longtrack A- and C-configuration VLA observations, resulting in a measurement of $\left\langle\theta_{\mathbf{M}}\right\rangle=0$ ". $167 \pm 0$ " 032 (with an rms scatter of 0 .'91), corresponding to a median linear size of $1.3 \pm 0.28 \mathrm{kpc}$ (with rms scatter $0.79 \mathrm{kpc}$ ). This was shown to match both the sizes of the dust emission regions measured at submillimeter wavelengths and the sizes measured in extinction-corrected $\mathrm{H} \alpha$ imaging.

Although consistent with the $1 \sigma$ measurement errors, and despite the high angular resolution of the $C$-HIGH images, our median linear size is larger than that measured by Murphy et al. (2017). This suggests that even half-arcsecond resolution is suboptimal for robust size measurements of high-redshift star- 

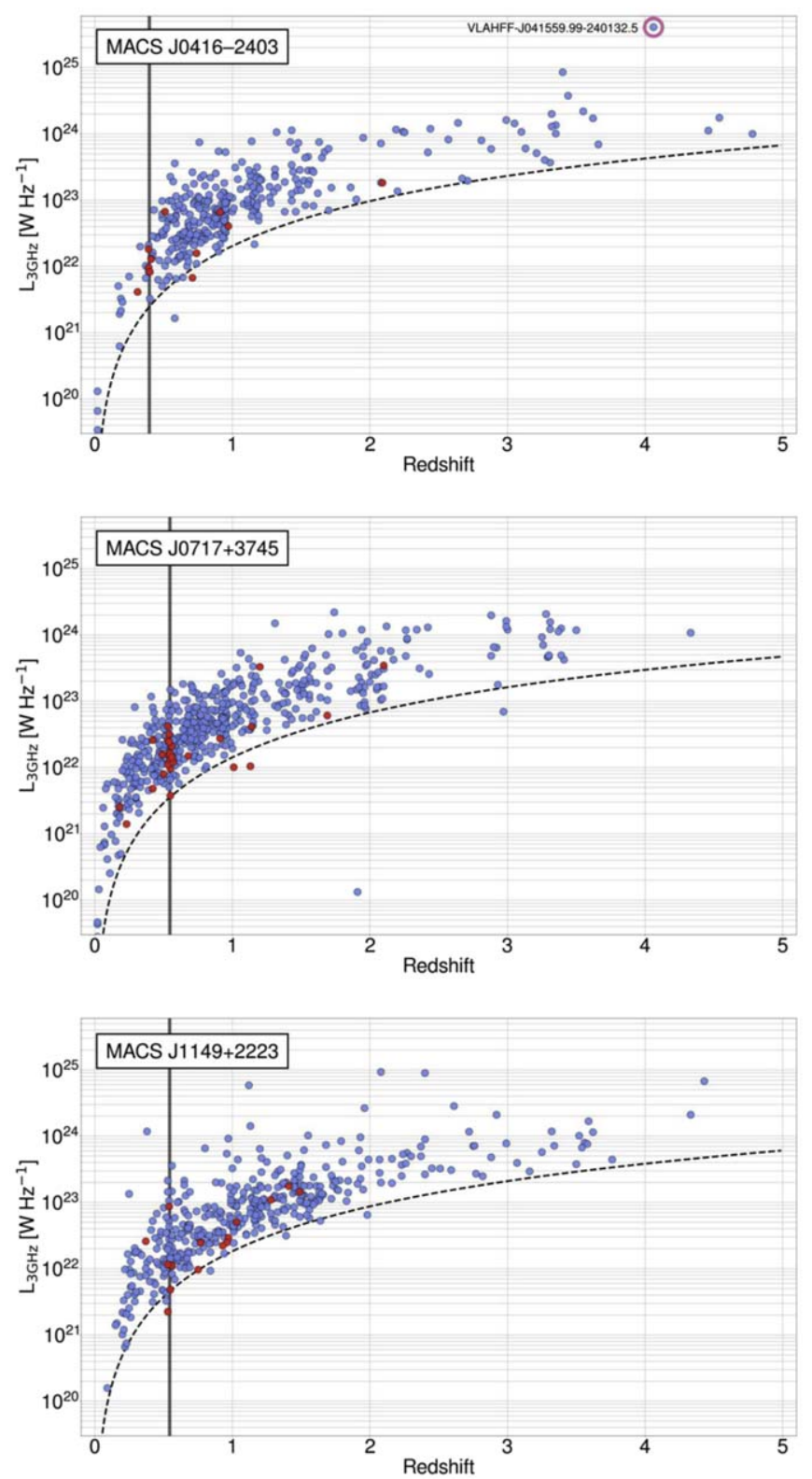

Figure 7. The $3 \mathrm{GHz}$ radio luminosities of the cataloged radio sources plotted against redshift for each of the three clusters. These have been demagnified according to the lensing magnification estimates in order to provide intrinsic luminosities. Sources with spectroscopic redshifts are marked in red. The vertical lines show the cluster redshifts. The dashed line shows the $5 \sigma$ detection limit based on the per-cluster $S$-HIGH noise measurements as provided in Table 2. Note that the extreme outlier in the MACS J0717 field is likely the result of an improper photometric redshift fit. The highlighted source is likely a newly discovered powerful radio galaxy at $z=4.06$.

forming galaxies at radio wavelengths, although a larger angular size is expected to be seen at lower radio frequencies due to cosmic-ray propagation effects. For a more detailed analysis of radio source sizes using our data, we again refer the reader to Jiménez-Andrade et al. (2021). The interferometers will not resolve out significant emission on these scales due to insufficient short spacings. Ignoring AGN contamination, the radio emission at both $6 \mathrm{GHz}$ and $10 \mathrm{GHz}$ should be arising from the star-forming regions. Given that the angular resolution of both of these studies exceeds that of Band 2 of SKA-MID $(0.95-1.76 \mathrm{GHz}), X$-band studies with the A configuration of the VLA are likely the best path to such intrinsic size
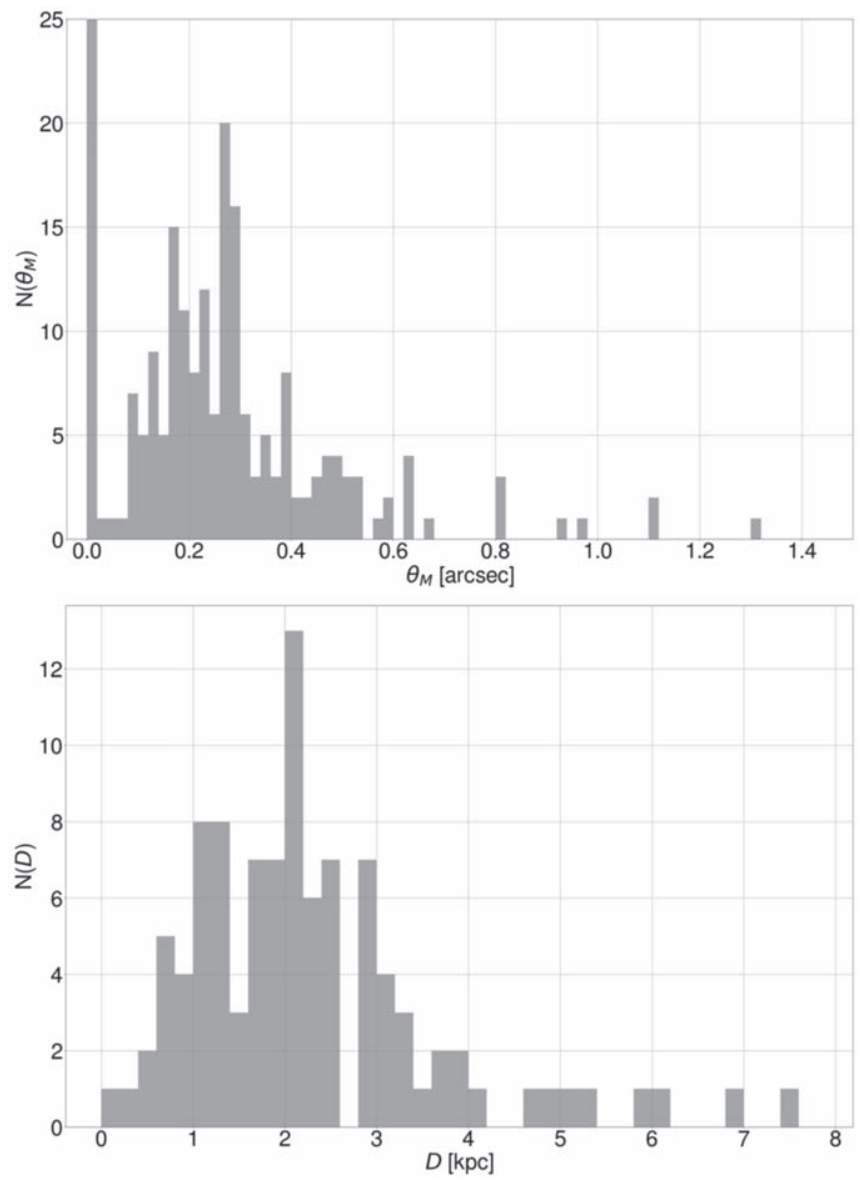

Figure 8. Distribution of the FWHM deconvolved source major axes as measured from the $C$-HIGH images (upper panel). The first bin (zero) contains 83 sources for which reliable deconvolved sizes could not be obtained. Not including these sources, the median deconvolved source size is 0.27 , with a median absolute deviation of 0 ". 1 . The lower panel shows the distribution for physical source sizes.

measurements prior to the arrival of the Next-Generation VLA (ngVLA; Murphy et al. 2018; McKinnon et al. 2019).

\subsection{Optical Colors of the Hosts of Compact Radio Sources}

Figure 9 shows the apparent magnitude differences of bands $B-z^{\prime}$ against $R-z^{\prime}$ from the Subaru catalogs. The hosts of compact radio sources are shown in blue. The rest of the entries in the Subaru catalogs are as a gray 2D histogram. The use of apparent instead of intrinsic magnitudes causes the significant scatter in the latter distribution; however, red (upper) and blue (lower) branches are evident. With reference also to Figure 3, the compact radio catalog is mostly detecting galaxies with redshifts higher than those of the clusters (although not necessarily on sight lines that pass through the mass distribution of the cluster). Despite the use of apparent magnitudes, the color distributions of the galaxies with radio detections are also visible. The radio data are deep enough to detect large numbers of galaxies in the blue cloud, i.e. regular star-forming spiral galaxies.

\subsection{The Radio Relic in MACS J1149.5+2223}

The diffuse radio emission associated with galaxy clusters is generally observed at low radio frequencies due to the low surface brightness and steep radio spectra; however, our $3 \mathrm{GHz}$ 

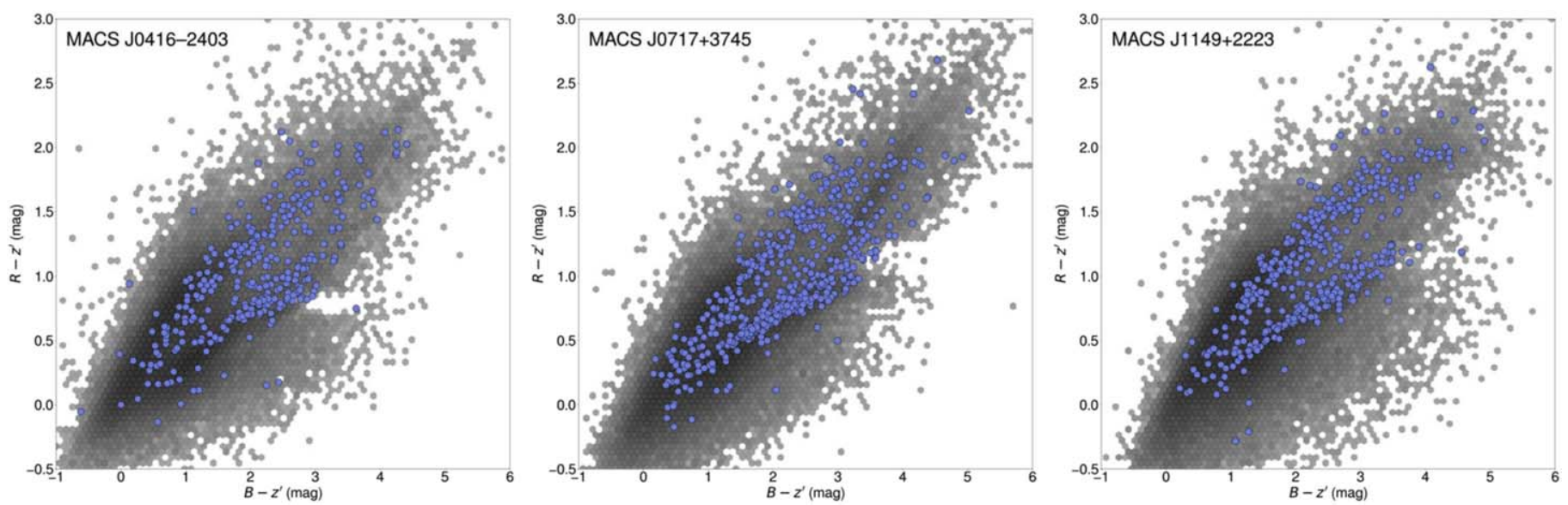

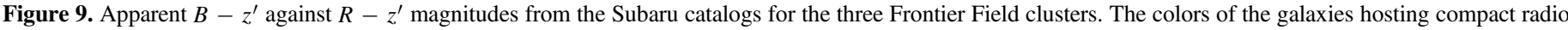

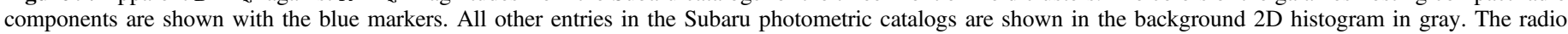
detections are representative of the general population of galaxies detected in the deep optical/near-infrared imaging.
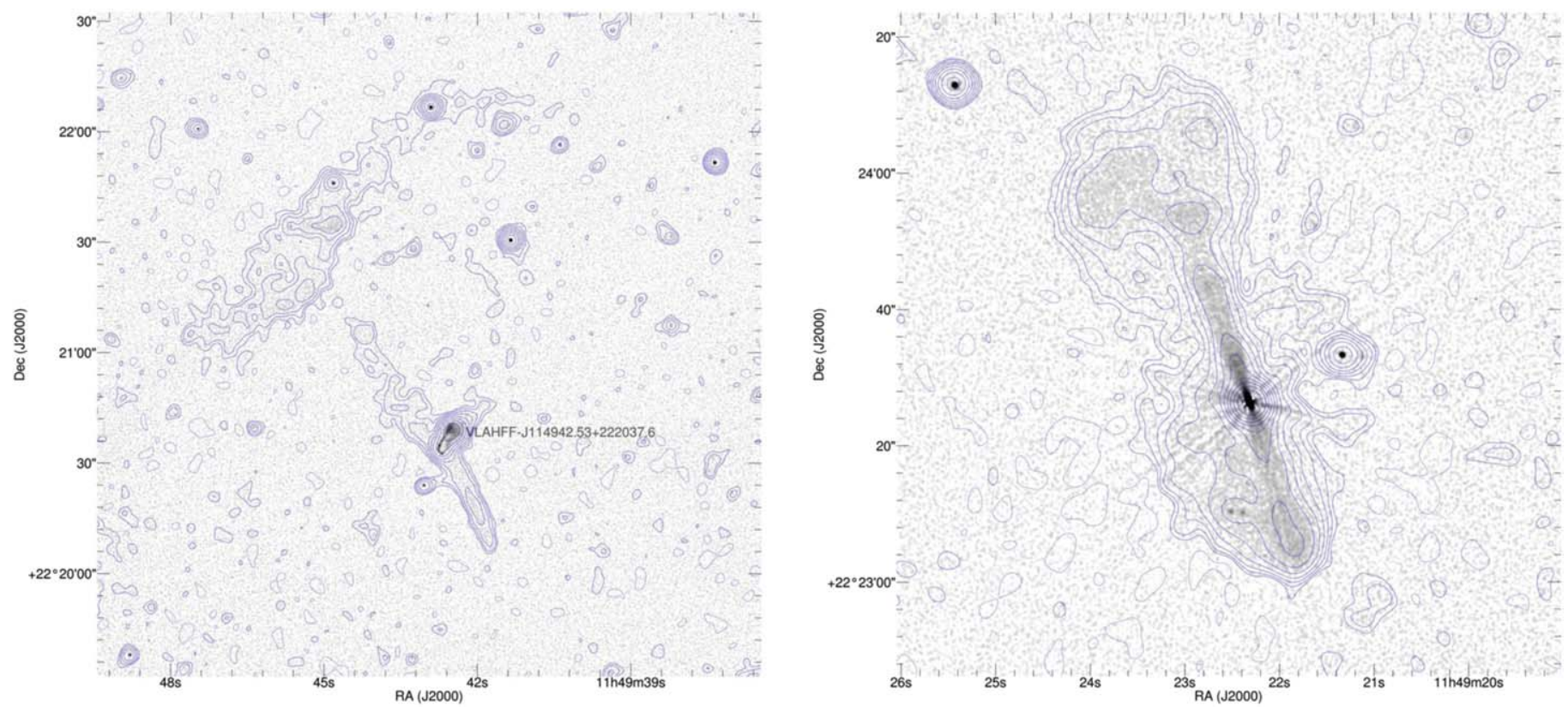

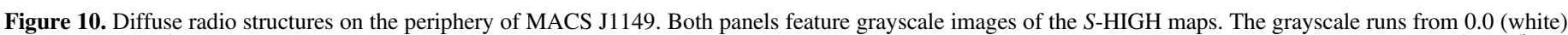

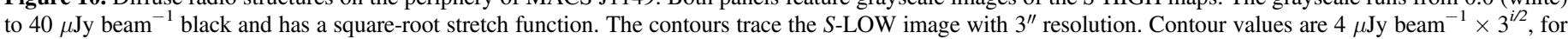

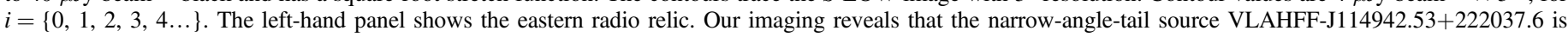

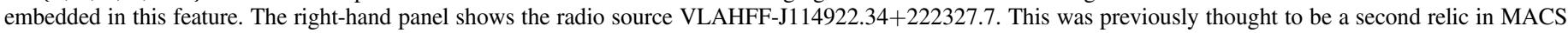
$\mathrm{J} 1149$, but is revealed to be an FR-I source at a lower redshift.

observations are deep enough to provide images of these structures at high angular resolution.

The central right-hand panels of Figures 1 and 2 are powerful illustrations of the usefulness of high angular resolution for relic and halo studies. MACS J0717 hosts one of the brightest radio halos known (e.g., van Weeren et al. 2017; Bonafede et al. 2018) and although the $S$-HIGH image resolves out the largest scales of the diffuse halo, the filamentary structures surrounding the central NAT radio galaxy can be seen with detail not possible with current lowfrequency instruments. The central tailed radio source has a host galaxy with a redshift that places it within the cluster; however, Rajpurohit et al. (2021) use spectral modeling to suggest that it is not interacting with the halo itself and is merely seen in projection.
Bonafede et al. (2012) present observations of MACS J1149 at $323 \mathrm{MHz}$ and $1.4 \mathrm{GHz}$, concluding the cluster hosts a double radio relic. Double relic structures are relatively rare, thought to arise when an ongoing cluster merger is seen from a favorable viewpoint (Bonafede et al. 2017). Figure 10 shows the view of the relevant regions afforded by our $3 \mathrm{GHz}$ data. The grayscale is the $S$-HIGH image, overlaid with the contours of the $3^{\prime \prime}$ resolution $S$-LOW image (please refer to the figure caption for further details). The left-hand panel shows the eastern relic, which is revealed to be a complex structure that is resolved into two distinct, almost perpendicular components. The bright peak seen at lower frequencies is actually an embedded NAT source (VLAHFF-J114922.34+222327.7, panel number 60 in Figure 13(a)). At $z=0.545$, this source could truly be embedded in the relic, rather than being a foreground or background source seen in projection. The right-hand panel 
shows the region of the reported second relic in MACS J1149, which in our high-resolution observations is revealed to be an FR-I source, VLAHFF-J114922.34+222327.7 (panel number 53 on Figure 13(a)). The high-resolution radio data also allow us to provide an unambiguous optical identification for the host galaxy, which has a photometric redshift of 0.24 , and so this entire radio structure is not actually associated with the cluster.

\section{Conclusions}

Using the VLA at 3 and $6 \mathrm{GHz}$, we have made some of the deepest $\left(\sim 1 \mu \mathrm{Jy}\right.$ beam $\left.{ }^{-1}\right)$, high-resolution $(\sim 0 . .5)$ radio images to date of the three MACS strong gravitational lensing clusters from the Frontier Fields program: MACS J0416.1 -2403, MACS J0717.5+3745, and MACS J1149.5+2223. From these images, we have derived catalogs with a total of 1966 compact radio components, 1296 of which have identified optical hosts. Relevant properties from the optical/nearinfrared data have been collated with the radio properties into a unified radio and optical/near-infrared catalog. We make use of the most recent mass and shear models available for the Frontier Fields to estimate the gravitational lensing magnification of the radio components with redshifts beyond those of the clusters. From this analysis, we detect a total of 13 moderately lensed $(2.1<\mu<6.5)$ sources. The optical/near-infrared colors of the radio detections show that we are detecting a significant population of regular, blue star-forming galaxies at high redshift.

The dual-frequency radio observations provide cataloged 3-6 GHz spectral index measurements for 169 compact components within the $50 \%$ level of the $C$-band primary beam. These are mostly dominated by sources with typical synchrotron spectra, although there is a significant population of flat and inverted spectrum sources. We also provide a catalog of intrinsic source sizes measured from the highest resolution $(\sim 0$ !! 3$) \mathrm{C}$-band images. Our median angular size of 0 ". $27 \pm 0$ ". 25 is somewhat larger than the 0 ". $167 \pm 0$ " 032 value measured at higher resolution by Murphy et al. (2017), and while our measurements offer good constraints, such work is likely better pursued using higher-frequency observations with the VLA's most extended configurations.

A total of 66 radio sources with extended morphologies were also identified, which are a mixture of sources hosting radio jets of numerous types, resolved spiral disks, and circumnuclear star formation. The advantage of using high angular resolution imaging for studies of the diffuse emission associated with galaxy clusters is exemplified by the detailed imaging of the diffuse emission associated with MACS J0717 and MACS J1149. In the case of the latter, we reveal that a bright feature in the radio relic previously observed at low frequencies is actually an embedded NAT source and that the putative second relic in this cluster is actually an FR-I-type radio galaxy at an intervening redshift.

The VLA Frontier Fields survey is a public legacy project, and we make all our catalog and image products freely available at https://science.nrao.edu/science/surveys/vla-ff.

We thank the anonymous referees for taking the time to read and comment on this paper. The National Radio Astronomy Observatory is a facility of the National Science Foundation operated under cooperative agreement by Associated Universities, Inc. Based on observations made with the NASA/ESA Hubble Space Telescope, obtained from the data archive at the
Space Telescope Science Institute. STScI is operated by the Association of Universities for Research in Astronomy, Inc. under NASA contract NAS 5-26555. Support for this work was provided by NASA through grant number HST-AR14306.001-A from the Space Telescope Science Institute, which is operated by AURA, Inc., under NASA contract NAS 5-26555. This work utilizes gravitational lensing models produced by PIs Brada, Natarajan \& Kneib (CATS), Merten \& Zitrin, Sharon, Williams, Keeton, Bernstein and Diego, and the GLAFIC group. This lens modeling was partially funded by the HST Frontier Fields program conducted by STScI. STScI is operated by the Association of Universities for Research in Astronomy, Inc. under NASA contract NAS 5-26555. The lens models were obtained from the Mikulski Archive for Space Telescopes (MAST). This work is based in part on data collected at the Subaru Telescope, which is operated by the National Astronomical Observatory of Japan. I.H. acknowledges support from the UK Science and Technology Facilities Council [ST/N000919/1]; the Oxford Hintze Centre for Astrophysical Surveys, which is funded through generous support from the Hintze Family Charitable Foundation; and the South African Radio Astronomy Observatory, which is a facility of the National Research Foundation (NRF), an agency of the Department of Science and Innovation.

Facilities: Karl G. Jansky Very Large Array, Hubble Space Telescope, Subaru Telescope.

Software: Astropy (Astropy Collaboration et al. 2013, 2018), CASA (McMullin et al. 2007), Matplotlib (Hunter 2007), ProFound (Robotham et al. 2018), PyBDSF (Mohan \& Rafferty 2015), wsclean (Offringa et al. 2014).

\section{Appendix A \\ Checking the Optical versus Radio Astrometric Frames}

Positions of compact radio components that are determined by the fitting of a Gaussian (or point) component to the pixel brightness distribution are subject to statistical uncertainties that are related to the resolution of the instrument as well as the $\mathrm{S} / \mathrm{N}$ of the detection (Condon 1997). In contrast, highresolution optical images may contain morphologies that may well be more complex than the radio. In this case, automated source extraction tends to emphasize the fitting of apertures that are appropriate for (often multiband) photometric measurements. The position of the galaxy then tends to be determined by using a brightness-weighted mean of the pixels within the aperture. Thus, even if the radio and optical emission have a cospatial origin, the automatically measured positions of the same object may differ slightly between the two wavelengths due to both the differing source extraction methods, as well as the statistical jitter that influences both with decreasing $\mathrm{S} / \mathrm{N}$ (as already introduced via Equation (1)). Additionally, radio and optical instruments may also have different absolute astrometric reference frames due to a variety of factors.

Figure 11 shows the checks we have performed to demonstrate that the above factors are not significant enough to influence the reliability of the cross-matching process, i.e., that the offsets in both an absolute and statistical sense are significantly smaller than the resolution element of the radio observations.

The difference between the radio and optical positions in both R.A. and decl. is determined for both the DeepSpace (red) and CLASH (blue) catalogs and plotted as a 2D histogram. In addition to this, the mean values per cluster and per catalog are 


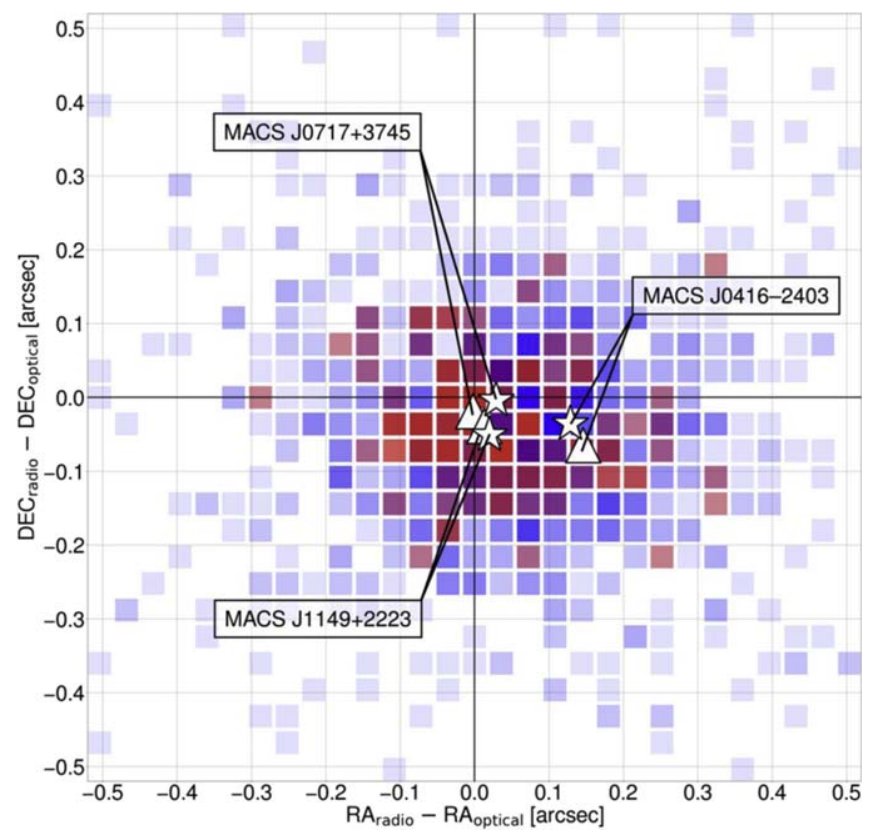

Figure 11. Differences between the radio and optical positions in R.A. and decl., for both the DeepSpace (red) and CLASH (blue) catalogs. The mean values of the offset distributions for the three clusters (as labeled) are also shown for both the DeepSpace (triangles) and CLASH (stars) catalogs. Note that the mean values are in all cases significantly smaller than the resolution element of the $S$-HIGH image, which was used as the starting point for generating the catalogs, as described in Section 3. The resolution of the $S$-HIGH images $\left(\sim 0.0^{\prime \prime} 5-1^{\prime \prime}\right.$ depending on the cluster decl.) is between half of the extent of the above figure and its full extent.

also plotted. In all cases, the mean offsets are smaller than the resolution element of the $S$-HIGH images used to extract the catalog.

\section{Appendix B \\ Deconvolved Source Sizes and Their Uncertainties when Observed with an Elliptical Beam}

In the case of a circular restoring beam, the deconvolved FWHM of a source is given by

$$
\theta_{\mathrm{M}}=\left(\phi^{2}-\theta_{\text {beam }}^{2}\right)^{1 / 2},
$$

where $\phi$ is the FWHM of the fitted component in the image, and $\theta_{\text {beam }}$ is the FWHM of the restoring beam. The error of $\theta_{M}$ is therefore

$$
\sigma_{\theta_{\mathrm{M}}}=\sigma_{\phi}\left[1-\left(\frac{\theta_{\text {beam }}}{\phi}\right)^{2}\right]^{-1 / 2} .
$$

In the case of an elliptical beam, estimating $\theta_{\mathrm{M}}$ and $\sigma_{\theta_{\mathrm{M}}}$ along the major axis of the source can be done with Equations (B1) and (B2) but only if the position angle of the restoring beam matches that of the source. To determine the deconvolved size $\left(\theta_{\mathrm{M}}^{\prime}\right)$ of a source along its major axis and the associated uncertainty $\left(\sigma_{\theta_{\mathrm{M}}}^{\prime}\right)$ in the general case, we have to determine the FWHM of the elliptical beam projected along the major axis of the source. This vector will be at a position angle $\theta_{\text {beam }}^{\prime}$; see Figure 12. The parametric equations of an ellipse are

$$
\begin{aligned}
x(t)= & a \cos (t) \cos \left[\operatorname{PAS}_{\mathrm{maj}}(\pi / 180)-(\pi / 2)\right] \\
& -b \sin (t) \sin \left[\operatorname{PAS}_{\mathrm{maj}}(\pi / 180)-(\pi / 2)\right]
\end{aligned}
$$

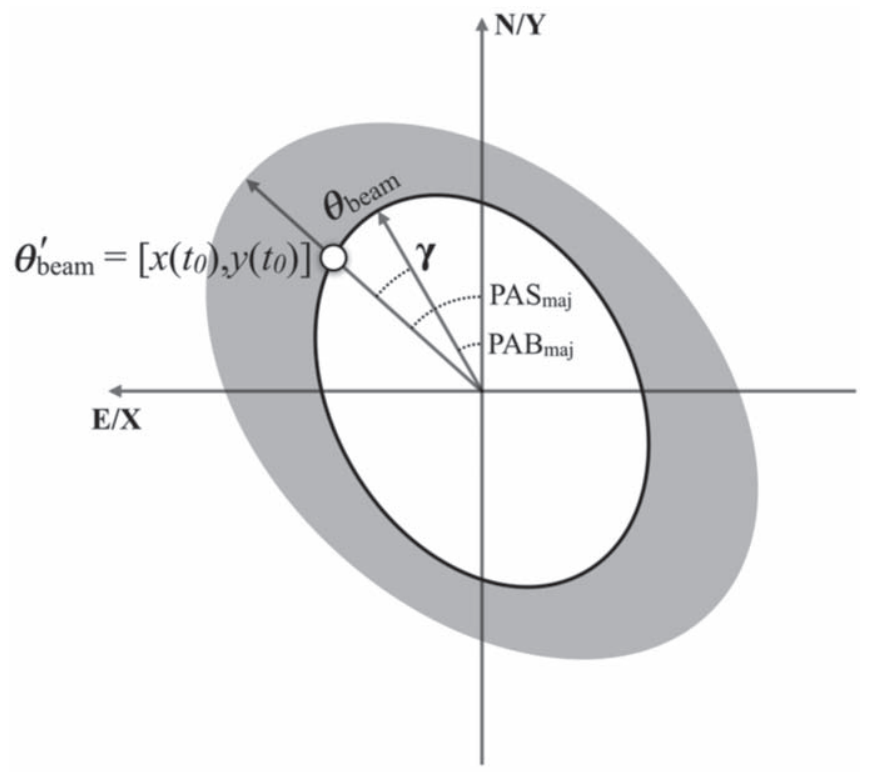

Figure 12. A 2D Gaussian radio source (the FWHM of which is represented by the gray ellipse) that has been observed with a $2 \mathrm{D}$ Gaussian restoring beam (the FWHM of which is the white ellipse). The arrows show the position angle of the FWHM major axis of the beam $\left(\mathrm{PAB}_{\mathrm{maj}}\right)$ and the source $\left(\mathrm{PAS}_{\mathrm{maj}}\right)$. Equations (B3) and (B4) can be evaluated at $t=t_{0}$ in order to infer the length of the beam along the direction of the source major axis $\left(\theta_{\text {beam }}^{\prime}\right)$ via Equation (B5).

and

$$
\begin{aligned}
y(t)= & a \cos (t) \sin \left[\operatorname{PAS}_{\mathrm{maj}}(\pi / 180)-(\pi / 2)\right] \\
& +b \sin (t) \cos \left[\operatorname{PAS}_{\mathrm{maj}}(\pi / 180)-(\pi / 2)\right],
\end{aligned}
$$

where $0 \leqslant t \leqslant 2 \pi$. If $a$ and $b$ are the FWHM major and minor axes of the source, then

$$
t=\arctan \left[\left(\frac{a}{b}\right) \tan (\gamma)\right]
$$

where $\gamma$ is the angle between the major axis of the ellipse and the vector to the point on the ellipse at $[x(t), y(t)]$. The vector on the beam ellipse that is aligned with the source major axis is therefore determined by evaluating Equations (B3)-(B5) for $\gamma=\mathrm{PAB}_{\text {maj }}-\mathrm{PAS}_{\mathrm{maj}}$, where all angles are given in degrees, and the standard convention of measuring position angles east of north applies. Once $x\left(t_{0}\right)$ and $y\left(t_{0}\right)$ are known, the FWHM of the elliptical beam along the source major axis is simply

$$
\theta_{\text {beam }}^{\prime}=\left[x\left(t_{0}\right)^{2}+y\left(t_{0}\right)^{2}\right]^{1 / 2},
$$

and its associated uncertainty is approximated as

$$
\sigma_{\theta_{\mathrm{M}}}^{\prime}=\sigma_{\phi^{\prime}}\left[1-\left(\frac{\theta_{\text {beam }}^{\prime}}{\phi^{\prime}}\right)^{2}\right]^{-1 / 2},
$$

where $\phi^{\prime}$ and $\sigma_{\phi^{\prime}}$ are the source major axis at the FWHM and its associated uncertainty, as measured by PyBDSF.

\section{Appendix C \\ Structure of the Compact Component Catalogs}

MACS J0416, MACS J0717, and MACS J1149 contain, respectively, 343, 535, and 418 radio sources that are well described by a point or Gaussian component and have 
associated optical identifications. These 1296 components each have entries in a per-cluster table, the columns of which are described below. Each cluster also has a table of radio components that do not have cataloged optical identifications within 1". For MACS J0416, MACS J0717, and MACS J1149, the total counts of radio components of this type are 162, 328, and 182 , correspondingly. These tables share columns 0-33 inclusive of the optically matched catalog.

(0): A unique positional identifier for each component of the form VLAHFF-JHHMMSS.SS \pm DDMMSS.S.

(1): The J2000 R.A. of the component in decimal degrees.

(2): The J2000 decl. of the component in decimal degrees.

(3) and (4): The $1 \sigma$ uncertainties in the position of the component in arcseconds. Note that these are uncertainties derived from the component fitting routines of PyBDSF, and as such do not include any systematic astrometric offsets.

(5) and (6): Integrated $S$-band flux density and $1 \sigma$ uncertainty in $\mu \mathrm{Jy}$.

(7) and (8): Peak $S$-band brightness and $1 \sigma$ uncertainty in $\mu \mathrm{Jy}_{\text {beam }}{ }^{-1}$.

(9) and (10): "Best" $S$-band flux density $\left(S^{*}\right)$ and $1 \sigma$ uncertainty in $\mu \mathrm{Jy}$ beam $^{-1}$. Please refer to Section 3.4 for further details.

(11): Assumed $S$-band primary beam correction factor that has been applied to columns (5)-(10) inclusive.

(12) and (13): Integrated $C$-band flux density and $1 \sigma$ uncertainty in $\mu \mathrm{Jy}$.

(14) and (15): Peak $C$-band brightness and $1 \sigma$ uncertainty in $\mu \mathrm{Jy} \mathrm{beam}^{-1}$.

(16) and (17): "Best" $C$-band flux density and $1 \sigma$ uncertainty in $\mu \mathrm{Jy}_{\text {beam }}{ }^{-1}$.

(18): Assumed $C$-band primary beam correction factor that has been applied to columns (12) to (17) inclusive.

(19) and (20): Spectral index $(\alpha)$ and $1 \sigma$ uncertainty for the component, derived from the $S$-band and matched-resolution $C$-band catalogs. Please refer to Section 4.2 for details.

(21)-(26): Major and minor axes (in arcseconds) and position angle (in degrees, east of north) of the fitted components in the $S$-HIGH images, together with the associated $1 \sigma$ uncertainties, as derived from the component fitting routines in PyBDSF. Values of 0.0 in these columns mean the component is unresolved in one or more dimensions.

(27) and (28): Major axis and associated uncertainty of the deconvolved components in the $S$-HIGH images. The major axis is the source finder's estimates of the intrinsic angular extent of the radio component; however, the uncertainties in the deconvolved size are determined using the method described in Section 3.4. As above, values of 0.0 in these columns mean the component is unresolved in one or more dimensions.

(29): A Boolean flag which is 1 if the source is deemed to be reliably resolved, 0 otherwise, based on the projection of the source major axis along the major axis of the restoring beam. See Section 3.4 for further details.

(30): ID of the assumed optical host galaxy in the DeepSpace (Shipley et al. 2018) catalogs. Note that in the DeepSpace catalogs these IDs begin from zero for each cluster, and so are not unique across all fields.

(31) and (32): R.A. and decl. in decimal degrees of the assumed optical host galaxy in the DeepSpace catalogs.

(33): Radial separation of the DeepSpace match in arcseconds.
(34): Spectroscopic redshift of the DeepSpace match, where available.

(35)-(37): Preferred photometric redshift and its lower and upper $68 \%$ confidence intervals of the DeepSpace match as returned by the EAZY code (Brammer et al. 2008), where available.

(38): Log of the stellar mass of the host galaxy $\left(M_{\odot}\right)$, as listed in the DeepSpace catalogs, derived using the FAST code (Kriek et al. 2018).

(39): Log of the star formation rate of the host galaxy $\left(M_{\odot}\right.$ $\mathrm{yr}^{-1}$ ), as listed in the DeepSpace catalogs, derived using the FAST code (Kriek et al. 2018).

(40): Log pf the specific star formation rate of the host galaxy $\left(M_{\odot} \mathrm{yr}^{-1}\right)$, as listed in the DeepSpace catalogs, derived using the FAST code (Kriek et al. 2018).

(41): ID of the assumed optical host galaxy in the CLASH catalogs.

(42) and (43): R.A. and decl. in decimal degrees of the assumed optical host galaxy in the CLASH catalogs.

(44): Radial separation of the CLASH match in arcseconds.

(45) and (46): CLASH Subaru z-band magnitude of the assumed host, and its uncertainty.

(47)-(49): Bayesian Photo-z (BPZ) redshift computed from the Subaru photometry, as well as the upper and lower limits. Please refer to Umetsu et al. (2014) and Medezinski et al. (2013) for details about the CLASH data, and the determination of photometric redshifts.

(50): Median lensing magnification value derived from the best available redshift. Preference is given to spectroscopic redshifts; however, the photometric values are used for the majority of objects where only such measurements are available.

(51): Median absolute deviation of the magnification values used to calculate column (51).

(52): The number of independent weak-lensing shear and mass surface density models used to compute the values in columns (50) and (51) inclusive. Values derived from several models tend to be closer to the high-magnification regions in the center of the cluster, covering the HST area. Please refer to Section 3.6 for details of the magnification calculations.

\section{Appendix D \\ Radio Sources with Extended Morphologies}

\section{D.1. Optical and Radio Overlays}

The three sections of Figure 13(a) show the $S$-HIGH contours for the 66 sources identified as having complex radio morphologies, overlaid on an RGB image formed from the Subaru $B-, R$-, and $z$-band images. Please refer to the caption of Figure 13(b) for further details. Note that these images are not primary beam corrected, in the interests of achieving a uniform contouring scheme, and are presented primarily for morphological classification. The properties of each of the complex sources are provided in order of decreasing total (primarybeam-corrected) flux density in Tables 4-6 for MACS J0416, MACS J0717, and MACS J1149, respectively. Here we provide brief comments on the radio morphology and optical host.

\section{D.2. Properties of Extended Radio Sources}

The properties of radio sources with extended morphologies are presented in Tables 4-6 for MACS J0416, MACS J0717, 


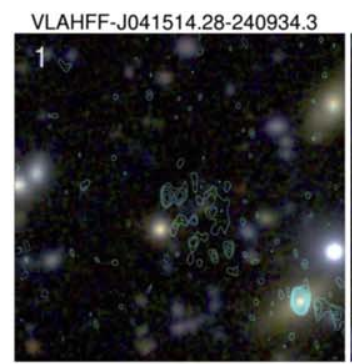

VLAHFF-J041528.24-240913.7
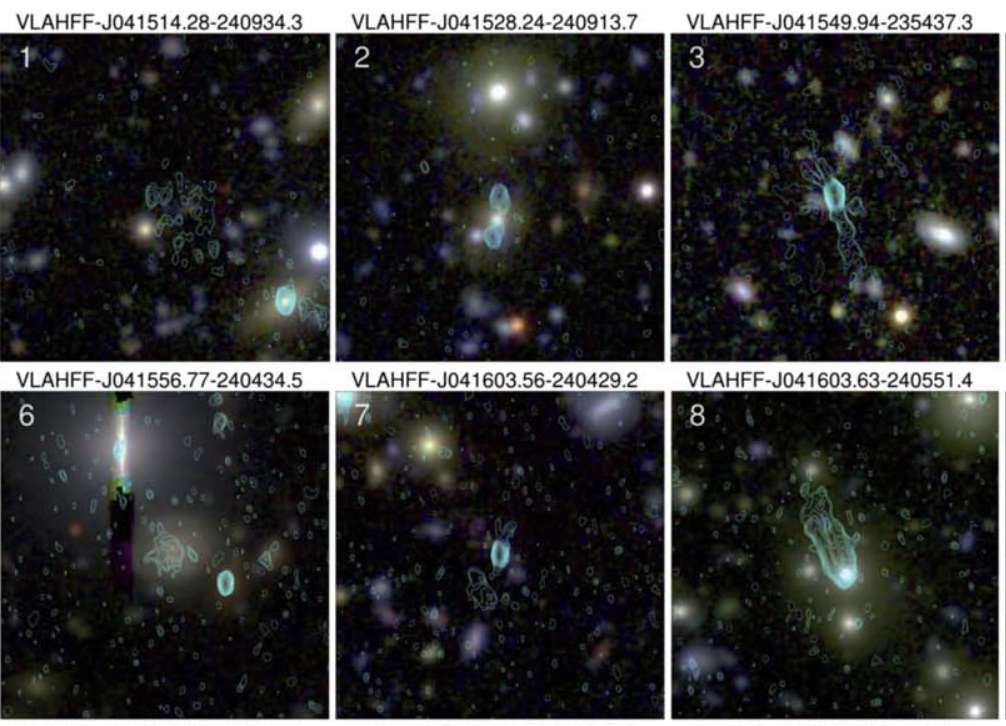

VLAHFF-J041553.04-240716.5

VLAHFF-J041554.13-240406.9

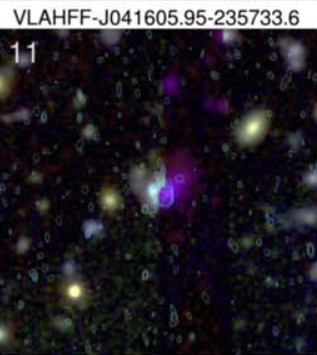

VLAHFF-J041609.06-240945.0
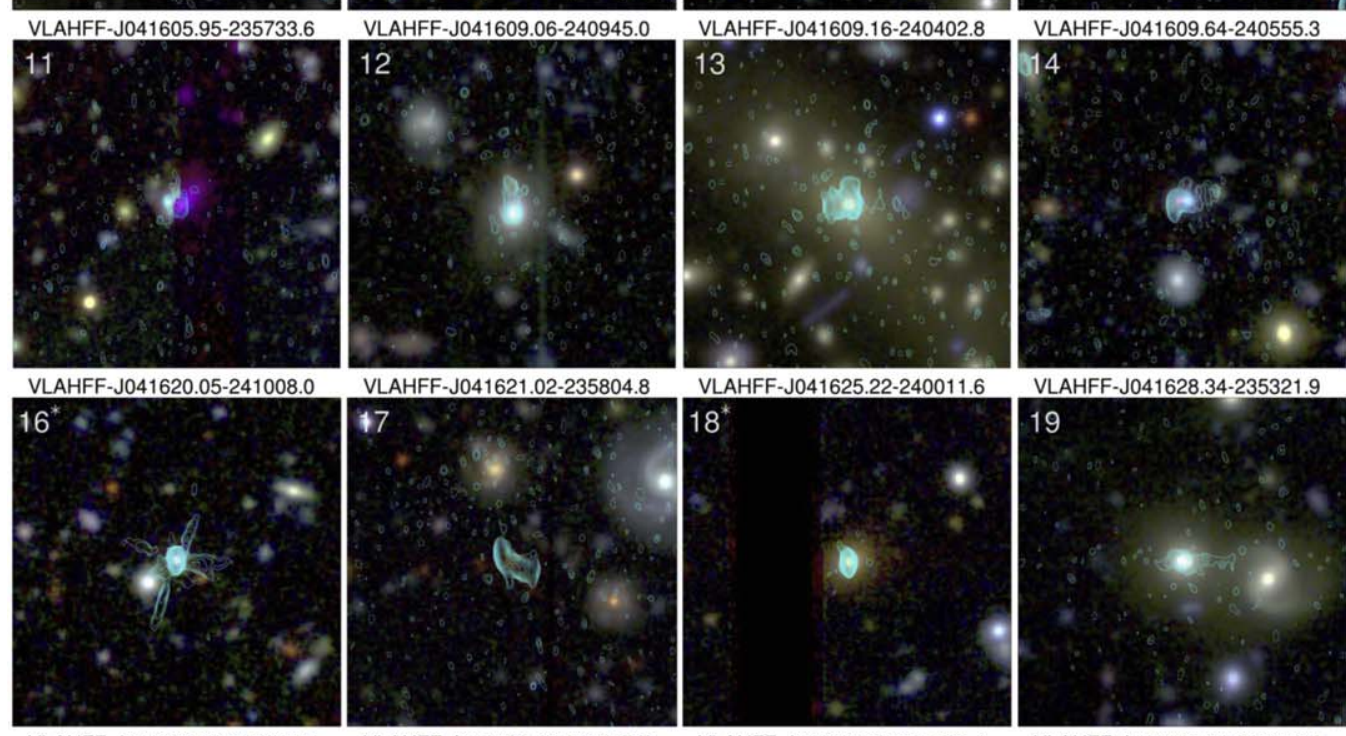

VLAHFF-J041628.34-235321.9
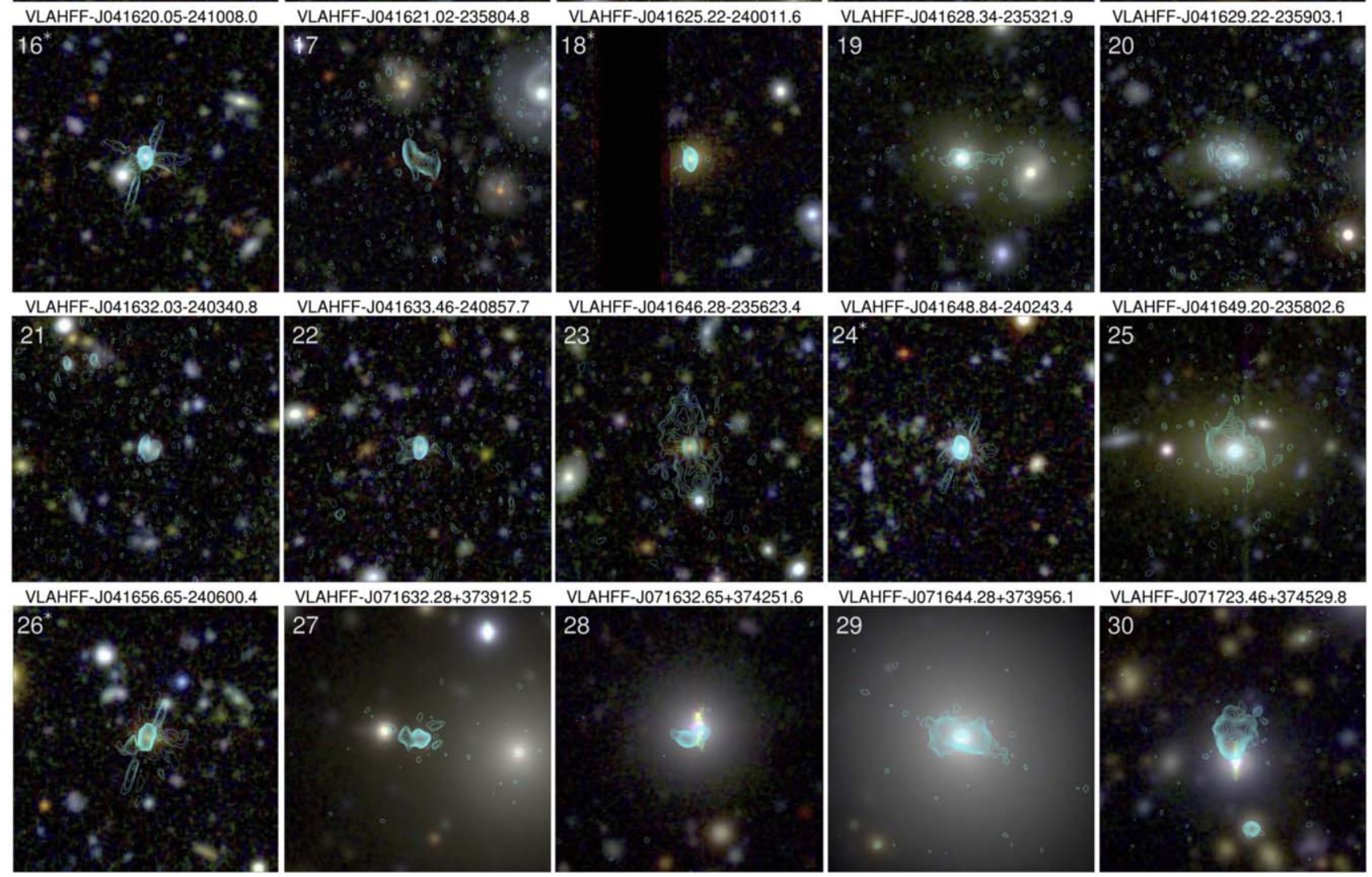

VLAHFF-J071632.28+373912.5
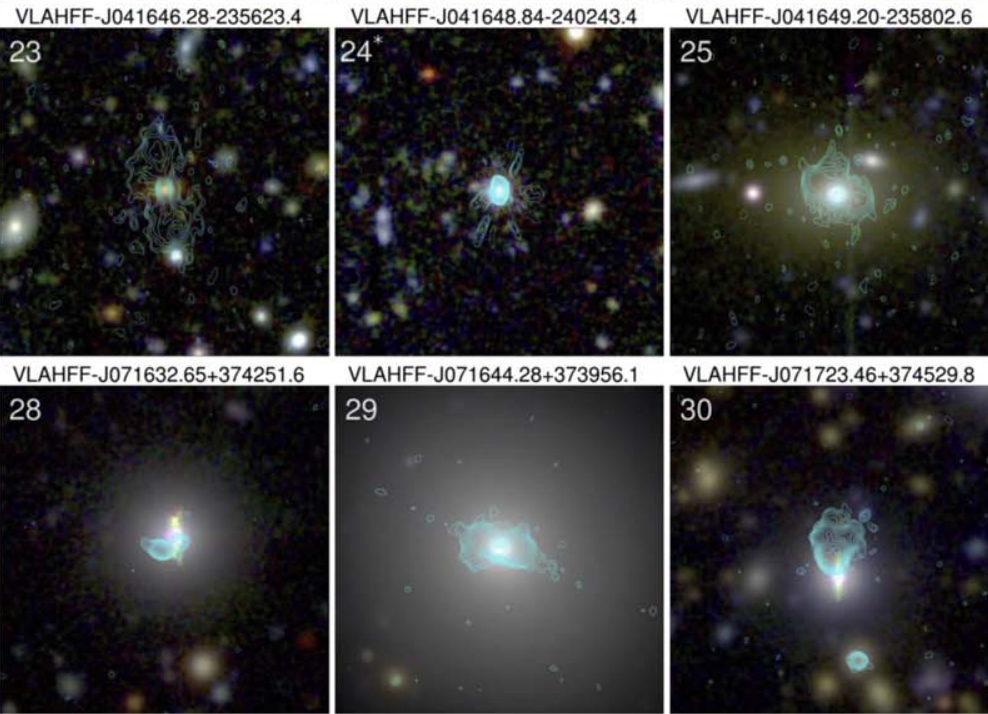

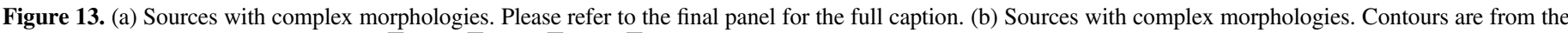

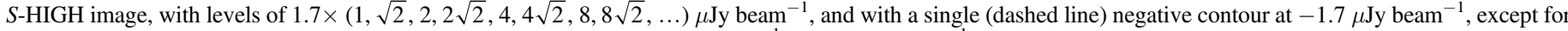

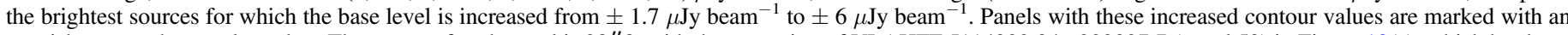

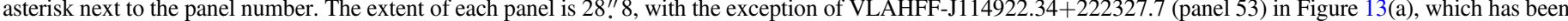

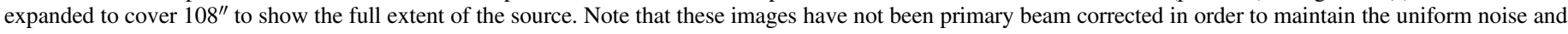

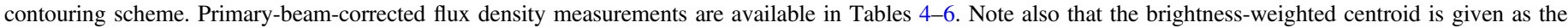
source position by ProFound and so the images may not necessarily be centered on the host galaxy. 


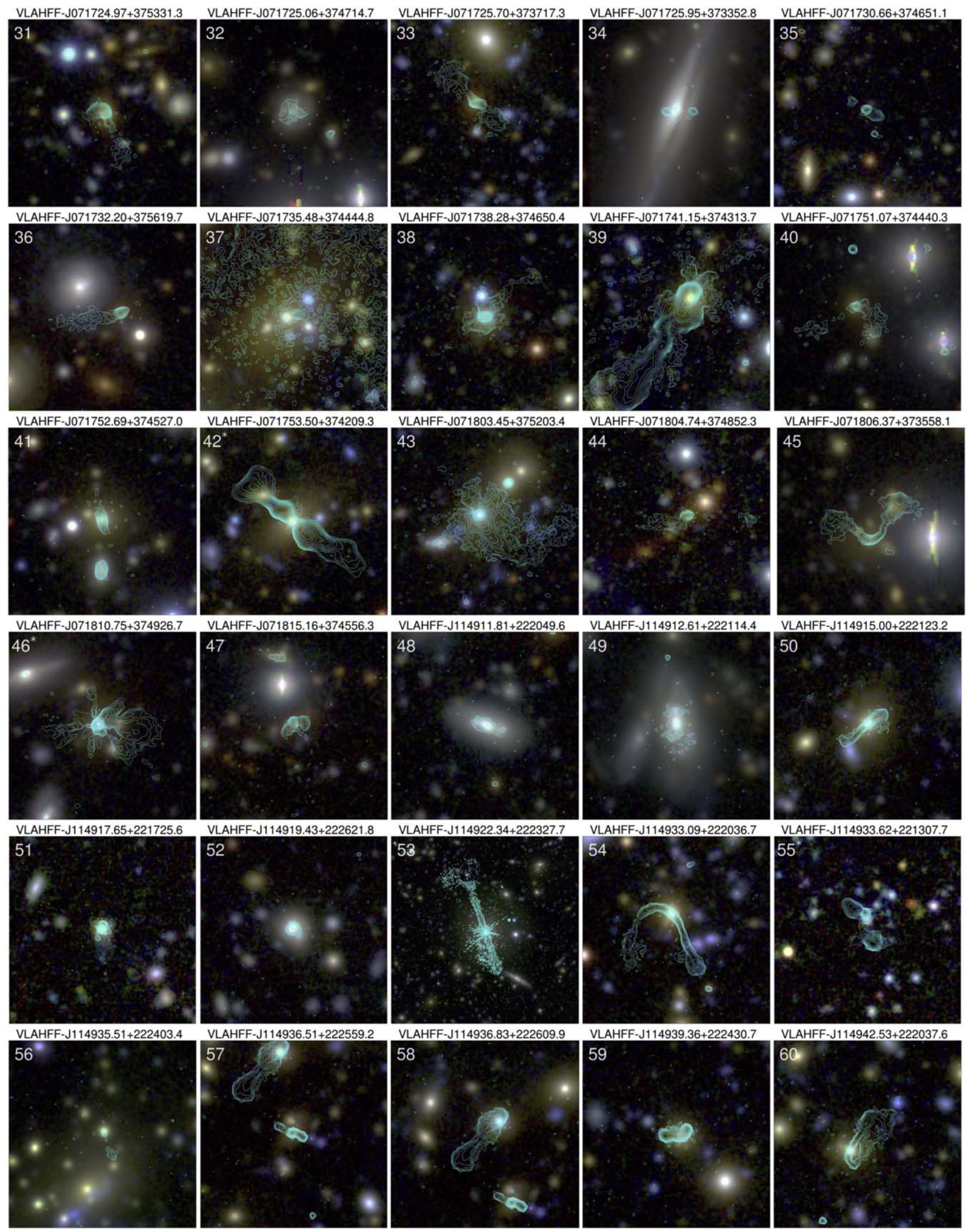

Figure 13. (Continued.)

and MACS J1149, respectively. The equatorial (J2000) positions are the flux-weighted centroids of the emission associated with each extended source, as determined by
ProFound. Optical IDs from the CLASH catalogs together with any available photometric redshifts are also listed, together with the integrated flux densities (and associated 


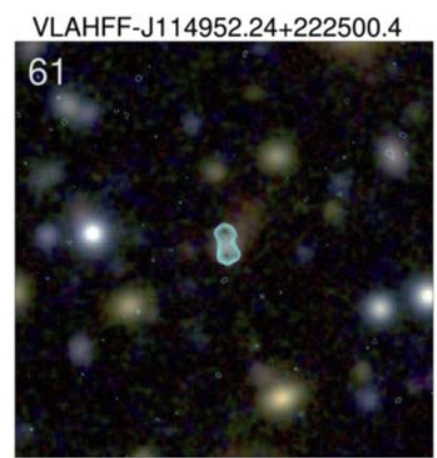

VLAHFF-J114957.22+222018.8

VLAHFF-J115003.87+221711.9
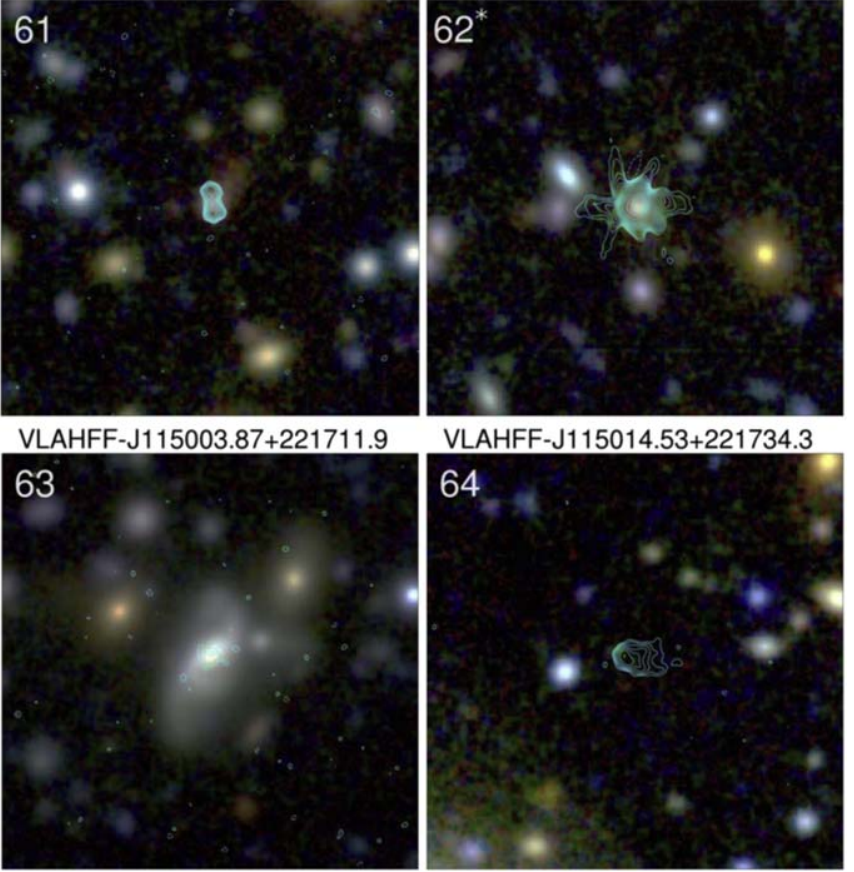

VLAHFF-J115014.53+221734.3

VLAHFF-J115015.28+222052.7
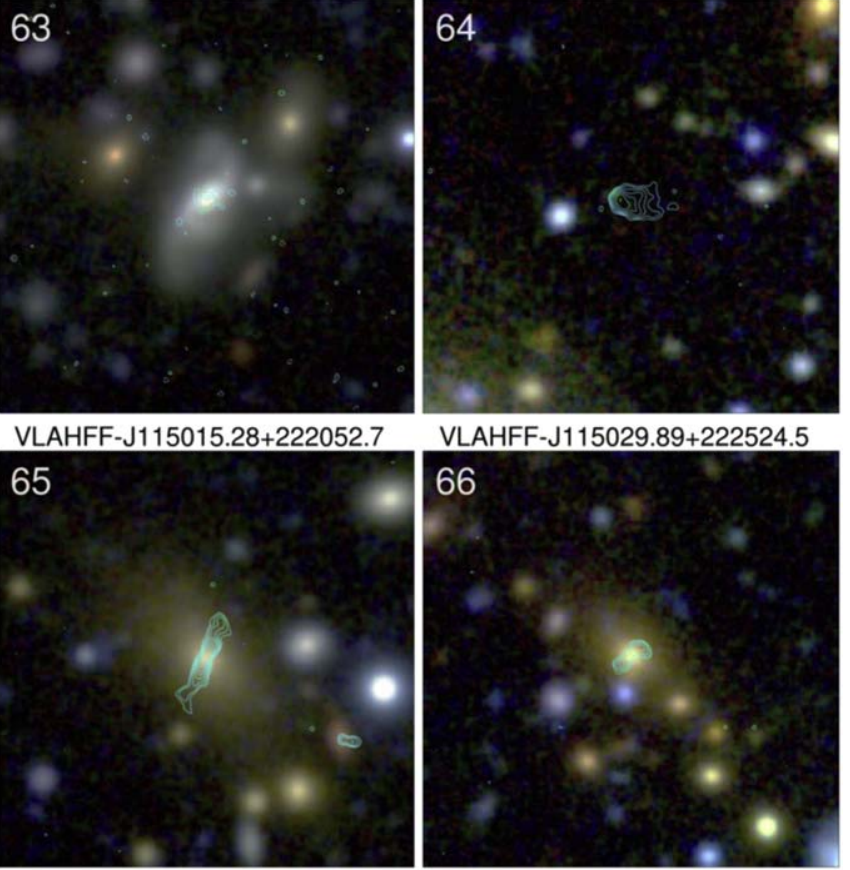

Figure 13. (Continued.)

uncertainty) as measured from the $S$-HIGH and $S$-LOW images. The latter provides a more robust estimate of the total integrated flux density of these sources at $3 \mathrm{GHz}$. As can be seen from the tables, the extended A configuration of the VLA (coupled with the weighting scheme required to deliver the high angular resolution required for the primary goal of these observations) resolves out a significant amount of extended emission.

\section{D.3. Notes on Individual Extended Sources}

(1) VLAHFF-J041514.28-240934.3: This is a low surface brightness isolated radio lobe, with a compact feature that may be a Fanaroff-Riley Type-2 (FR-II; Fanaroff \& Riley 1974) hotspot. It is associated with the compact core in the lower right of the panel, which also hosts a lobe to the west; however, this source is not cataloged as it falls outside the primary beam cutoff. The host appears to be an elliptical.

(2) VLAHFF-J041528.24-240913.7: Double-lobed structure associate with an elliptical galaxy. The host galaxy has no cataloged optical/near-infrared ID.

(3) VLAHFF-J041549.94-235437.3: FR-I (Fanaroff \& Riley 1974) radio source. The host is not visible in the Subaru imaging, so is possibly dust obscured.
(4) VLAHFF-J041553.04-240716.5: This is an FR-I structure, or possibly due to the low axial ratio of the lobes, a relic radio galaxy. No compact hot spots are evident. The host is an elliptical with a redshift of 0.499 .

(5) VLAHFF-J041554.13-240406.9: Two optical galaxies are enveloped by edge-brightened radio emission with a diffuse tail structure. The tabulated host is the central galaxy, at a redshift of 1.645 .

(6) VLAHFF-J041556.77-240434.5: This radio source shows resolved emission apparently associated with a spiral galaxy at $z=1.695$. The radio emission appears to be asymmetric with respect to the disk, so it may be a jet structure associated with a rare spiral AGN, rather than star-formationdriven radio emission. An alternative explanation is that the alignment with the spiral is a projection effect, and this is a radio lobe, possibly associated with the compact radio source associated with the elliptical galaxy also seen in this panel.

(7) VLAHFF-J041603.56-240429.2: A possible hybrid morphology source, the radio emission appears to be FR-Ilike on the northern side, with a diffuse radio lobe on the southern side. No hot spots are visible. The optical host is a $z=0.945$ galaxy with unclear or disturbed optical morphology.

(8) VLAHFF-J041603.63-240551.4: This is an NAT radio galaxy, hosted by an elliptical galaxy at $z=0.392$. Tailed radio 
Table 4

Positions and Integrated Flux Densities of the Extended Radio Sources in MACS J0416

\begin{tabular}{|c|c|c|c|c|c|c|c|c|}
\hline ID & $\begin{array}{l}\text { R.A. } \\
\text { (deg) }\end{array}$ & $\begin{array}{l}\text { Decl. } \\
\text { (deg) }\end{array}$ & CLASH ID & $z_{\mathrm{BPZ}}$ & $\begin{array}{l}\begin{array}{l}S_{\text {int }}^{\text {HIGH }} \\
(\mathrm{mJy})\end{array}\end{array}$ & $\begin{array}{c}\sigma S_{\text {int }}^{\mathrm{HIGH}} \\
(\mathrm{mJy})\end{array}$ & $\begin{array}{l}S_{\text {int }}^{\text {LOW }} \\
(\mathrm{mJy})\end{array}$ & $\begin{array}{c}\sigma S_{\text {int }}^{\mathrm{LOW}} \\
(\mathrm{mJy})\end{array}$ \\
\hline VLAHFF-J041514.28-240934.3 & 63.80952 & -24.15953 & $\ldots$ & $\ldots$ & 2.6 & 0.05 & $\ldots$ & $\cdots$ \\
\hline VLAHFF-J041528.24-240913.7 & 63.86767 & -24.15382 & $\cdots$ & $\cdots$ & 3.14 & 0.07 & 17.26 & 0.17 \\
\hline VLAHFF-J041553.04-240716.5 & 63.97102 & -24.12125 & 43053 & 0.499 & 14.09 & 0.12 & 86.8 & 0.13 \\
\hline VLAHFF-J041554.13-240406.9 & 63.97557 & -24.0686 & 53515 & 1.645 & 33.52 & 0.03 & 103.55 & 0.05 \\
\hline VLAHFF-J041556.77-240434.5 & 63.98655 & -24.07626 & 54833 & 1.695 & 1.2 & 0.03 & 16.9 & 0.08 \\
\hline VLAHFF-J041604.63-240415.0 & 64.01931 & -24.07086 & 53930 & 0.037 & 4.76 & 0.03 & 13.78 & 0.04 \\
\hline VLAHFF-J041604.84-241028.0 & 64.0202 & -24.17446 & 32639 & 0.288 & 16.91 & 0.02 & 53.73 & 0.06 \\
\hline VLAHFF-J041605.95-235733.6 & 64.0248 & -23.95933 & 77422 & 0.273 & 2.05 & 0.06 & 4.56 & 0.06 \\
\hline VLAHFF-J041609.06-240945.0 & 64.03779 & -24.16253 & 33689 & 0.025 & 2.07 & 0.03 & 6.58 & 0.06 \\
\hline VLAHFF-J041609.16-240402.8 & 64.03821 & -24.06745 & 56589 & 0.438 & 12.62 & 0.02 & 40.61 & 0.05 \\
\hline VLAHFF-J041609.64-240555.3 & 64.04018 & -24.09871 & 47184 & 0.945 & 2.86 & 0.04 & 7.08 & 0.04 \\
\hline VLAHFF-J041628.34-235321.9 & 64.11809 & -23.88944 & 99186 & 0.358 & 11.14 & 0.26 & 57.44 & 0.55 \\
\hline VLAHFF-J041629.22-235903.1 & 64.12177 & -23.9842 & 72655 & 0.574 & 2.6 & 0.05 & 6.48 & 0.06 \\
\hline VLAHFF-J041632.03-240340.8 & 64.13346 & -24.06134 & 55131 & 0.874 & 2.26 & 0.03 & 7.54 & 0.06 \\
\hline VLAHFF-J041633.46-240857.7 & 64.13944 & -24.14937 & 35525 & 1.184 & 9.85 & 0.03 & 32.74 & 0.08 \\
\hline VLAHFF-J041646.28-235623.4 & 64.19284 & -23.93984 & 81280 & 3.53 & 164.61 & 1.1 & 272.8 & 0.42 \\
\hline VLAHFF-J041648.84-240243.4 & 64.20352 & -24.04541 & 58828 & 0.831 & 131.98 & 0.08 & 482.07 & 0.3 \\
\hline VLAHFF-J041649.20-235802.6 & 64.20504 & -23.9674 & 77823 & 0.423 & 41.31 & 0.28 & 141.28 & 0.29 \\
\hline VLAHFF-J041656.65-240600.4 & 64.23607 & -24.10013 & 46835 & 1.148 & 295.60 & 0.37 & 1109.11 & 0.78 \\
\hline
\end{tabular}

Note. Unique identifiers with the same format as described in Appendix $\mathrm{C}$ are based on the flux-weighted centroids of the radio emission as identified by the ProFound source finder. Optical host IDs from the CLASH data are provided, along with photometric redshift estimates where available. Integrated flux density measurements (and associated uncertainties) are provided from both the $S$-HIGH and $S$-LOW images, with the latter providing a more robust estimate of the total integrated flux density at $3 \mathrm{GHz}$.

galaxies are very common in massive clusters, and the host galaxy redshift associates it with the MACS J0416 cluster.

(9) VLAHFF-J041604.63-240415.0: A comparatively lowredshift $(z=0.037)$ elliptical galaxy, likely hosting a one-sided core-jet structure.

(10) VLAHFF-J041604.84-241028.0: Another one-sided core-jet structure, hosted by an elliptical galaxy at $z=0.288$. There are some artifacts associated with this source; however, we believe the jet structure to be real, due to its three-contour significance, and the lack of correspondingly bright positive or negative features at the expected $120^{\circ}$ positions that one would expect from a PSF-like artifact in a VLA image.

(11) VLAHFF-J041605.95-235733.6: There is an issue with the green channel in the optical imaging across this source; however, it appears to be a pair of merging or conjunction of two galaxies. The radio emission exhibits a double peak and a tail.

(12) VLAHFF-J041609.06-240945.0: This is another comparatively low-redshift source $(z=0.025)$. The radio morphology exhibits two peaks; however, it is most probably a corejet structure associated with the coincident elliptical galaxy.

(13) VLAHFF-J041609.16-240402.8: A double-peaked radio source with a diffuse envelope, coincident with an elliptical galaxy with a photometric redshift of 0.438 . The two radio peaks are of comparable brightness, however only one of them is coincident with an optical peak, and the detected radio structure is small compared to the size of the host. This could be a young radio jet or a double AGN.
(14) VLAHFF-J041609.64-240555.3: This source has what appears to be a one-sided core-jet structure; however, it could also be a young FR-II source as the innermost radio peak is offset from the peak of the optical emission.

(15) VLAHFF-J041613.94-235645.0: This relatively lowredshift $(z=0.024)$ massive elliptical exhibits a feature in the optical imaging that resembles a spiral arm, possibly a tidal tail. The radio emission is a compact core with a diffuse envelope, possibly driven by both a central AGN and circumnuclear star formation.

(16) VLAHFF-J041620.05-241008.0: A strong compact radio source associated with a galaxy at $z=1.176$. The image is dynamic range limited at this position. The linear diagonal features are likely residual sidelobes from the imperfect deconvolution; however, the diffuse FR-I-like jet structure to the west may be real.

(17) VLAHFF-J041621.02-235804.8: A FR-I type galaxy, or possible wide-angle tail (WAT) source at $z=1.438$.

(18) VLAHFF-J041625.22-240011.6: A strong radio source associated with the core of an elliptical at $z=0.516$. The source exhibits a possible core-jet extension, but is dynamic range limited.

(19) VLAHFF-J041628.34-235321.9: FR-I radio morphology associated with an elliptical galaxy at $z=0.358$.

(20) VLAHFF-J041629.22-235903.1: Resolved radio emission associated with the disk of a spiral galaxy at $z=0.574$.

(21) VLAHFF-J041632.03-240340.8: A core-jet source or possibly a resolved disk at $z=0.874$ 
Table 5

Positions and Integrated Flux Densities of the Extended Radio Sources in MACS J0717

\begin{tabular}{|c|c|c|c|c|c|c|c|c|}
\hline ID & $\begin{array}{l}\text { R.A. } \\
\text { (deg) }\end{array}$ & $\begin{array}{l}\text { Decl. } \\
\text { (deg) }\end{array}$ & CLASH ID & $z_{\mathrm{BPZ}}$ & $\begin{array}{c}S_{\mathrm{int}}^{\mathrm{HIGH}} \\
(\mathrm{mJy})\end{array}$ & $\begin{array}{c}\sigma S_{\text {int }}^{\mathrm{HIGH}} \\
(\mathrm{mJy})\end{array}$ & $\begin{array}{l}S_{\text {int }}^{\mathrm{LOW}} \\
(\mathrm{mJy})\end{array}$ & $\begin{array}{c}\sigma S_{\text {int }}^{\mathrm{LOW}} \\
(\mathrm{mJy})\end{array}$ \\
\hline VLAHFF-J071632.28+373912.5 & 109.1345 & 37.65348 & 26801 & 2.813 & 301.87 & 0.34 & 396.62 & 0.47 \\
\hline VLAHFF-J071632.65+374251.6 & 109.13604 & 37.71434 & $\cdots$ & $\cdots$ & 25.63 & 0.14 & 89.57 & 0.19 \\
\hline VLAHFF-J071723.46+374529.8 & 109.34777 & 37.75829 & $\cdots$ & $\cdots$ & 23.85 & 0.03 & 68.43 & 0.03 \\
\hline VLAHFF-J071724.97+375331.3 & 109.35408 & 37.89205 & 71157 & 0.626 & 24.64 & 0.08 & 56.79 & 0.12 \\
\hline VLAHFF-J071725.06+374714.7 & 109.35442 & 37.78742 & 54902 & 0.431 & 1.8 & 0.03 & 4.09 & 0.03 \\
\hline VLAHFF-J071730.66+374651.1 & 109.37778 & 37.78089 & 49833 & 1.524 & 2.51 & 0.05 & 6.12 & 0.05 \\
\hline VLAHFF-J071732.20+375619.7 & 109.38419 & 37.93882 & 86311 & 0.882 & 24.46 & 0.2 & 69.94 & 0.24 \\
\hline VLAHFF-J071735.48+374444.8 & 109.39787 & 37.7458 & 44706 & 0.555 & 1.06 & 0.03 & 12.51 & 0.14 \\
\hline VLAHFF-J071738.28+374650.4 & 109.40954 & 37.78067 & 50671 & 0.605 & 48.96 & 0.05 & 69.63 & 0.04 \\
\hline VLAHFF-J071741.15+374313.7 & 109.42149 & 37.72047 & 39947 & 0.56 & 214.0 & 0.72 & 631.53 & 0.11 \\
\hline VLAHFF-J071751.07+374440.3 & 109.46282 & 37.74454 & 44825 & 0.537 & 6.82 & 0.06 & 13.55 & 0.06 \\
\hline VLAHFF-J071806.37+373558.1 & 109.52657 & 37.59949 & $\cdots$ & $\cdots$ & 141.17 & 0.44 & 130.6 & 0.20 \\
\hline VLAHFF-J071810.75+374926.7 & 109.54479 & 37.82411 & 59007 & 0.642 & 396.47 & 0.68 & 1320.51 & 0.84 \\
\hline VLAHFF-J071815.16+374556.3 & 109.56318 & 37.76565 & 48526 & 0.906 & 6.78 & 0.05 & 19.25 & 0.07 \\
\hline
\end{tabular}

Note. Please refer to the caption of Table 4 for further details.

(22) VLAHFF-J041633.46-240857.7: One-sided core-jet structure at a redshift of 1.184 .

(23) VLAHFF-J041646.28-235623.4: FR-II radio galaxy at $z=3.53$.

(24) VLAHFF-J041648.84-240243.4: Another bright core with a possible jet extension, but the image is dynamic range limited. The host galaxy is at a redshift of 0.831 .

(25) VLAHFF-J041649.20-235802.6: A double-lobed radio galaxy at $z=0.423$. Host optical morphology is elliptical, with a possible merging counterpart.

(26) VLAHFF-J041656.65-240600.4: Bright compact core, with possible FR-I structure but the image is dynamic range limited. The host galaxy is at $z=1.148$.

(27) VLAHFF-J071632.28+373912.5: Core-jet radio source associated with a compact elliptical galaxy at $z=2.813$.

(28) VLAHFF-J071632.65+374251.6: This is likely a corejet source, but due to the offset radio peak, we assume that the true optical host is obscured by a bright foreground object at $z=0.017$.

(29) VLAHFF-J071644.28+373956.1: Resolved radio emission from an elliptical galaxy at $z=0.076$, or possibly a compact FR-I source associated with the nucleus of the host.

(30) VLAHFF-J071723.46+374529.8: This source is an NAT radio galaxy. The compact core is prominent in the $C$ HIGH image; however, there is no compact component at that position in the $S$-HIGH image indicating that the core is synchrotron self-absorbed. The optical host is obscured by the foreground galaxy or star in Subaru imaging. The radio source visible to the south in the corresponding figure is unrelated.

(31) VLAHFF-J071724.97+375331.3: A highly asymmetric twin jet structure, or possibly a WAT with a resolved out or otherwise nondetected jet. The optical data place the host at $z=0.626$.

(32) VLAHFF-J071725.06+374714.7: Resolved radio emission associated with a spiral galaxy at $z=0.431$. The radio morphology shows a possible spiral arm structure.
(33) VLAHFF-J071725.70+373717.3: Diffuse double-lobed radio source at $z=0.709$, with no clear sign of the hotspot emission at the lobe heads.

(34) VLAHFF-J071725.95+373352.8: An intriguing source at redshift 0.067 , showing compact jets associated with either a large edge-on spiral, or an elliptical with a prominent dust lane.

(35) VLAHFF-J071730.66+374651.1: This source is likely a compact FR-II radio galaxy at $z=1.524$.

(36) VLAHFF-J071732.20+375619.7: A tailed radio galaxy, or extremely one-sided source. At a redshift of 0.882 , it is not an NAT or WAT associated with the foreground cluster.

(37) VLAHFF-J071735.48+374444.8: A compact core-jet source, seen to be embedded in the bright halo emission of the MACS0717 cluster. With a photometric redshift of 0.555 , the host galaxy is likely to be a cluster member.

(38) VLAHFF-J071738.28+374650.4: A FR-I source at $z=0.605$. The northern jet has a significant extension that changes direction with respect to the jet emission close to the core; however, there is no southern counterpart to this very extended feature.

(39) VLAHFF-J071741.15+374313.7: This source is an NAT with twisted jets that are clearly visible in the $C$-HIGH image. The host appears to be an elliptical galaxy with a photometric redshift of 0.56 .

(40) VLAHFF-J071751.07+374440.3: A WAT source at $z=0.537$, likely associated with a cluster member.

(41) VLAHFF-J071752.69+374527.0: This is a compact FR-I source at $z=0.502$.

(42) VLAHFF-J071753.50+374209.3: A double-lobed source with a pair of diffuse inner hot spots, possibly a restarted AGN. The host is at $z=0.563$.

(43) VLAHFF-J071803.45+375203.4: A head-tail radio galaxy at $z=0.4$ with very diffuse, low axial ratio jet emission. Could possibly be an NAT or WAT seen in projection. 
Table 6

Positions and Integrated Flux Densities of the Extended Radio Sources in MACS J1149

\begin{tabular}{|c|c|c|c|c|c|c|c|c|}
\hline ID & $\begin{array}{l}\text { R.A. } \\
\text { (deg) }\end{array}$ & $\begin{array}{l}\text { Decl. } \\
\text { (deg) }\end{array}$ & CLASH ID & $z_{\mathrm{BPZ}}$ & $\begin{array}{l}S_{\text {int }}^{\mathrm{HIGH}} \\
(\mathrm{mJy})\end{array}$ & $\begin{array}{c}\sigma S_{\mathrm{int}}^{\mathrm{HIGH}} \\
(\mathrm{mJy})\end{array}$ & $\begin{array}{l}S_{\text {int }}^{\text {LOW }} \\
(\mathrm{mJy})\end{array}$ & $\begin{array}{c}\sigma S_{\text {int }}^{\mathrm{LOW}} \\
(\mathrm{mJy})\end{array}$ \\
\hline VLAHFF-J114911.81+222049.6 & 177.29924 & 22.34713 & 47811 & 0.107 & 3.07 & 0.05 & 11.64 & 0.05 \\
\hline VLAHFF-J114912.61+222114.4 & 177.30256 & 22.354 & 52412 & 0.175 & 3.57 & 0.06 & 19.8 & 0.08 \\
\hline VLAHFF-J114917.65+221725.6 & 177.32358 & 22.29046 & 34122 & 0.708 & 27.24 & 0.03 & 63.5 & 0.08 \\
\hline VLAHFF-J114919.43+222621.8 & 177.33099 & 22.4394 & 68009 & 0.191 & 1.48 & 0.02 & 6.672 & 0.05 \\
\hline VLAHFF-J114922.34+222327.7 & 177.34311 & 22.39104 & 62337 & 0.24 & 1.80 & 0.03 & $\ldots$ & $\ldots$ \\
\hline VLAHFF-J114935.51+222403.4 & 177.39796 & 22.40095 & 58850 & 0.553 & 0.49 & 0.02 & 2.09 & 0.04 \\
\hline VLAHFF-J114936.51+222559.2 & 177.40217 & 22.43313 & 65767 & 0.739 & 5.37 & 0.02 & 74.12 & 0.05 \\
\hline VLAHFF-J114936.83+222609.9 & 177.40349 & 22.43609 & 67239 & 0.563 & 13.49 & 0.04 & 74.21 & 0.06 \\
\hline VLAHFF-J114939.36+222430.7 & 177.41402 & 22.40853 & 60954 & 0.561 & 17.16 & 0.02 & 69.36 & 0.03 \\
\hline VLAHFF-J114942.53+222037.6 & 177.42725 & 22.34378 & 46364 & 0.545 & 7.77 & 0.04 & $\ldots$ & $\cdots$ \\
\hline VLAHFF-J114952.24+222500.4 & 177.46767 & 22.4168 & 62088 & 1.123 & 1.48 & 0.02 & 6.27 & 0.03 \\
\hline VLAHFF-J115029.89+222524.5 & 177.62458 & 22.42348 & 64436 & 0.848 & 5.49 & 0.18 & 28.8 & 0.29 \\
\hline
\end{tabular}

Note. Please refer to the caption of Table 4 for further details.

(44) VLAHFF-J071804.74+374852.3: Possible hybrid morphology (FR-I/FR-II) radio source at $z=0.955$.

(45) VLAHFF-J071806.37+373558.1: A bent tail (NAT/ WAT) radio source with a clear optical host in the Subaru images but no cataloged counterpart.

(46) VLAHFF-J071810.75+374926.7: This source is possibly a one-sided FR-I with a large opening angle, but the image is dynamic range limited due to the bright radio core. The host galaxy is at $z=0.642$.

(47) VLAHFF-J071815.16+374556.3: The radio emission shows either a core-jet source, or possibly the resolved disk of the optical host galaxy at $z=0.906$.

(48) VLAHFF-J114911.81+222049.6: This radio source is likely driven by circumnuclear star formation in an elliptical galaxy at $z=0.107$.

(49) VLAHFF-J114912.61+222114.4: Resolved radio emission associated with the disk of a spiral galaxy at $z=0.175$.

(50) VLAHFF-J114915.00+222123.2: FR-I structure associated with a redshift 0.488 elliptical galaxy.

(51) VLAHFF-J114917.65+221725.6: Double compact radio sources, both within the redshift 0.708 host. Possible double nucleus.

(52) VLAHFF-J114919.43+222621.8: The morphology of this source suggests it is resolved star-formation-driven radio emission at $z=0.191$.

(53) VLAHFF-J114922.34+222327.7: This is a bright FR-I radio source on the periphery of the MACS1149 field; however, with a photometric redshift of 0.24 it is likely not associated with the cluster itself. The host appears to be a large, low surface brightness elliptical galaxy. We discuss this source further in Section 4.7.

(54) VLAHFF-J114933.09+222036.7: This source is a WAT with a redshift of 0.558 , likely associated with the MACS J1149 cluster.

(55) VLAHFF-J114933.62+221307.7: There is no cataloged counterpart for the host galaxy of this source; however, a strong candidate is visible in Subaru imaging. The radio morphology is a disturbed, asymmetric FR-II structure with prominent radio plumes.

(56) VLAHFF-J114935.51+222403.4: This object is a onesided core-jet source associated with a redshift 0.553 galaxy, likely a cluster member.

(57) VLAHFF-J114936.51+222559.2: A compact FR-II source with a $z=0.739$ host galaxy.

(58) VLAHFF-J114936.83+222609.9: A tailed radio galaxy, likely an NAT or WAT seen in projection. The optical counterpart is clear, with a cataloged redshift of 0.563 , likely associating it with the MACS J1149 cluster.

(59) VLAHFF-J114939.36+222430.7: This appears to be a bent FR-I source at $z=0.536$.

(60) VLAHFF-J114942.53+222037.6: Another NAT at $z=0.545$. This source appears to be embedded in the eastern radio relic associated with the MACS1149 cluster. See Section 4.7 for more details.

(61) VLAHFF-J114952.24+222500.4: Three components are visible in this compact source, which is possibly a young FR-II with a redshift of 1.123 .

(62) VLAHFF-J114957.22+222018.8: The radio emission from this strong source appears to be resolved in multiple directions, but the image is dynamic range limited at this point, so this must be interpreted with care. The host galaxy is at a redshift of 0.986 .

(63) VLAHFF-J115003.87+221711.9: This is diffuse radio emission at low $\mathrm{S} / \mathrm{N}$. Likely driven by star formation, it is associated with the core of a spiral galaxy at $z=0.229$.

(64) VLAHFF-J115014.53+221734.3: No optical counterpart is visible for this source. Given the morphology, it is likely to be an isolated radio lobe. The morphology also suggests the most likely core counterpart is the compact source VLAHFF$\mathrm{J} 115008.52+221733.8(z=0.25$, and not visible in this panel). At this redshift, the projected separation is $328 \mathrm{kpc}$, which is not unreasonable for an FR-II. No western counterpart lobe is visible on the other side of the putative core. 
(65) VLAHFF-J115015.28+222052.7: This is an FR-I source associated with an elliptical galaxy at $z=0.54$, likely associated with MACS J1149.

(66) VLAHFF-J115029.89+222524.5: The radio emission is aligned perpendicular to the major axis of the elliptical host galaxy $(z=0.848)$ and is likely a compact pair of radio jets.

\section{ORCID iDs}

I. Heywood (1) https://orcid.org/0000-0001-6864-5057

E. J. Murphy (1) https://orcid.org/0000-0001-7089-7325

E. F. Jiménez-Andrade (1) https://orcid.org/0000-00022640-5917

L. Armus (1) https://orcid.org/0000-0003-3498-2973

C. DeCoursey (1) https://orcid.org/0000-0002-4781-9078

M. Dickinson (1) https://orcid.org/0000-0001-5414-5131

E. Momjian (ib https://orcid.org/0000-0003-3168-5922

I. Smail (1) https://orcid.org/0000-0003-3037-257X

\section{References}

Astropy Collaboration, Price-Whelan, A. M., Sipőcz, B. M., et al. 2018, AJ, 156,123

Astropy Collaboration, Robitaille, T. P., Tollerud, E. J., et al. 2013, A\&A, 558, A33

Bonafede, A., Brüggen, M., van Weeren, R., et al. 2012, MNRAS, 426, 40 Bonafede, A., Brüggen, M., Rafferty, D., et al. 2018, MNRAS, 478, 2927

Bonafede, A., Cassano, R., Brüggen, M., et al. 2017, MNRAS, 470, 3465

Brammer, G. B., van Dokkum, P. G., \& Coppi, P. 2008, ApJ, 686, 1503

Briggs, D. S. 1995, AAS, 187, 112

Caminha, G. B., Grillo, C., Rosati, P., et al. 2017, A\&A, 600, A90

Castellano, M., Amorín, R., Merlin, E., et al. 2016, A\&A, 590, A31

Condon, J. J. 1997, PASP, 109, 166

Delvecchio, I., Smolčić, V., Zamorani, G., et al. 2017, A\&A, 602, A3

Di Criscienzo, M., Merlin, E., Castellano, M., et al. 2017, A\&A, 607, A30

Diego, J. M., Broadhurst, T., Zitrin, A., et al. 2015, MNRAS, 451, 3920

Dudzevičiūtè, U., Smail, I., Swinbank, A. M., et al. 2020, MNRAS, 494, 3828

Ebeling, H., Edge, A. C., \& Henry, J. P. 2001, ApJ, 553, 668

Ebeling, H., Ma, C.-J., \& Barrett, E. 2014, ApJS, 211, 21

Fanaroff, B. L., \& Riley, J. M. 1974, MNRAS, 167, 31P

Gim, H. B., Yun, M. S., Owen, F. N., et al. 2019, ApJ, 875, 80

Hale, C. L., Robotham, A. S. G., Davies, L. J. M., et al. 2019, MNRAS, 487, 3971

Heywood, I., Hale, C. L., Jarvis, M. J., et al. 2020, MNRAS, 496, 3469

Heywood, I., Jarvis, M. J., Baker, A. J., et al. 2016, MNRAS, 460, 4433
Hunter, J. D. 2007, CSE, 9, 90

Huynh, M. T., Bell, M. E., Hopkins, A. M., Norris, R. P., \& Seymour, N. 2015, MNRAS, 454, 952

Huynh, M. T., Seymour, N., Norris, R. P., \& Galvin, T. 2020, MNRAS, 491, 3395

Ivison, R. J., Greve, T. R., Dunlop, J. S., et al. 2007, MNRAS, 380, 199

Jackson, N. 2011, ApJL, 739, L28

Jauzac, M., Richard, J., Limousin, M., et al. 2016, MNRAS, 457, 2029

Jiménez-Andrade, E. F., Murphy, E. J., Heywood, I., et al. 2021, ApJ, submitted

Kawamata, R., Oguri, M., Ishigaki, M., Shimasaku, K., \& Ouchi, M. 2016, ApJ, 819, 114

Kriek, M., van Dokkum, P. G., Labbé, I., et al. 2018, FAST: Fitting and Assessment of Synthetic Templates, Astrophysics Source Code Library, ascl: 1803.008

Limousin, M., Richard, J., Jullo, E., et al. 2016, A\&A, 588, A99

Lotz, J. M., Koekemoer, A., Coe, D., et al. 2017, ApJ, 837, 97

Magnelli, B., Popesso, P., Berta, S., et al. 2013, A\&A, 553, A132

McKinnon, M., Beasley, A., Murphy, E., et al. 2019, BAAS, 51, 81

McMullin, J. P., Waters, B., Schiebel, D., Young, W., \& Golap, K. 2007, adass XVI, 376, 127

Medezinski, E., Umetsu, K., Nonino, M., et al. 2013, ApJ, 777, 43

Merlin, E., Amorín, R., Castellano, M., et al. 2016, A\&A, 590, A30

Merlin, E., Fontana, A., Ferguson, H. C., et al. 2015, A\&A, 582, A15

Mohan, N., \& Rafferty, D. 2015, PyBDSF: Python Blob Detection and Source Finder, Astrophysics Source Code Library, ascl:1502.007

Murphy, E. J., Bolatto, A., Chatterjee, S., et al. 2018, ASPC, 517, 3

Murphy, E. J., Chary, R.-R., Dickinson, M., et al. 2011, ApJ, 732, 126

Murphy, E. J., Momjian, E., Condon, J. J., et al. 2017, ApJ, 839, 35

Offringa, A. R., McKinley, B., Hurley-Walker, N., et al. 2014, MNRAS, 444,606

Ogrean, G. A., van Weeren, R. J., Jones, C., et al. 2015, ApJ, 812, 153

Perley, R. 2016, EVLA Memo 195, VLA Expansion Project Memoranda, https://library.nrao.edu/public/memos/evla/EVLAM_195.pdf

Rajpurohit, K., Wittor, D., van Weeren, R. J., et al. 2021, A\&A, 646, A56

Robotham, A. S. G., Davies, L. J. M., Driver, S. P., et al. 2018, MNRAS, 476, 3137

Schmidt, K. B., Treu, T., Brammer, G. B., et al. 2014, ApJL, 782, L36

Shipley, H. V., Lange-Vagle, D., Marchesini, D., et al. 2018, ApJS, 235, 14

Singh, V., Beelen, A., Wadadekar, Y., et al. 2014, A\&A, 569, A52

Smolčić, V., Novak, M., Bondi, M., et al. 2017, A\&A, 602, A1

Umetsu, K., Medezinski, E., Nonino, M., et al. 2014, ApJ, 795, 163

van Weeren, R. J., Röttgering, H. J. A., Brüggen, M., \& Cohen, A. 2009, A\&A, 505, 991

van Weeren, R. J., Ogrean, G. A., Jones, C., et al. 2016, ApJ, 817, 98

van Weeren, R. J., Ogrean, G. A., Jones, C., et al. 2017, ApJ, 835, 197

Vanzella, E., Fontana, A., Zitrin, A., et al. 2014, ApJL, 783, L12

Wang, T., Schreiber, C., Elbaz, D., et al. 2019, Natur, 572, 211 